\title{
A finite element model for magnetohydrodynamic squeeze-film flows
}

\author{
Jordan R. Wagner and C. Fred Higgs III \\ Department of Mechanical Engineering, Rice University, 6100 Main St., Houston, Texas 77005, USA
}

(Received 5 October 2018; accepted 23 November 2018; published online 20 December 2018)

\begin{abstract}
A computational model is developed to analyze magnetohydrodynamic (MHD) squeeze-film flows featuring an electrically conducting fluid subjected to imposed magnetic and electric fields. The model is based on the so-called MHD Reynolds equation for squeeze-films-an extension of the classical hydrodynamic Reynolds equation. A complete derivation of the MHD Reynolds equation is performed by applying thin-film and quasi-steady assumptions to the Maxwell/Navier-Stokes system coupled by the Lorentz force. The resulting equation is a two-dimensional and variable-coefficient Poisson equation for pressure, which reduces to the purely hydrodynamic form in the limit of vanishing Hartmann number. A geometric calculus formulation facilitates the reduction of the mathematical system into two dimensions, which is a challenge in standard vector calculus due to the cross product. The model permits realistic geometrical representations of the constraining squeeze-surfaces, and we demonstrate the use of a multi-variate Weierstrass-Mandelbrot fractal to numerically generate scale-invariant surface roughness profiles. Ultimately, the governing equation is solved with the Galerkin finite element method. Several numerical examples are conducted to highlight some of the model's capabilities. MHD forces - as well as the roughness, geometry, and topology of the squeeze surfaces-are shown to significantly influence flow characteristics. Published by AIP Publishing. https://doi.org/10.1063/1.5064598
\end{abstract}

\section{INTRODUCTION}

Squeeze-film flows emanate from approaching surfaces that are initially separated by a thin film of fluid. The converging surfaces squeeze the interfacial fluid and, in effect, a hydrodynamic pressure is developed that tends to damp the incident motion of the surfaces. This phenomenon is often exploited by natural (e.g., synovial joints) and engineering systems. From an engineering standpoint, squeeze-film dampers are used to control vibrations in a wide range of mechanical systems, such as in micro-electro-mechanical systems (MEMS), ${ }^{1,2}$ accelerometers, ${ }^{3}$ and turbojet aircraft engines. ${ }^{4}$ The purely hydrodynamic squeeze-film problem has a rich history dating back to nearly a decade before Osbourne Reynolds delivered his famous 1886 paper on the theory of lubrication. ${ }^{5}$ In his seminal work, Reynolds linearized the Navier-Stokes $(\mathrm{N}-\mathrm{S})$ equations with a thin-film assumption and integrated over the film-thickness to attain a two-dimensional, Poisson equation for the hydrodynamic pressure developed in the fluid.

The load-carrying capacity of the fluid is a critical design factor in engineering and is largely dictated by viscous forces, so an interfacial fluid of adequate viscosity is essential. However in high-temperature applications, the reduction in viscosity brings about a decline in performance; in extreme conditions, conventional lubricants are likely to be rendered ineffective. For this reason, liquid metals have been used in nuclear and space engineering applications due to their ability to maintain viscosity at high temperatures. ${ }^{6}$ Additionally since these fluids are good electrical conductors, they are amenable to manipulation with electromagnetic forces. Consequently, there has been recent interest in leveraging magnetohydrodynamic (MHD) effects to preserve the performance of squeeze-film bearings in such extreme environments. $^{7,8}$ MHD squeeze-films would also allow for in situ, precise control of damping properties by simple alteration of the imposed fields, which is not feasible with purely hydrodynamic squeeze-films.

The study of MHD informally began in 1832, when Michael Faraday attempted to measure the voltage induced across the River Thames as it flowed through Earth's magnetic field. ${ }^{9}$ The two primary processes involved in MHD are electrodynamics, governed by the Maxwell equations, and hydrodynamics, governed by the N-S equations. These two dynamical equation systems are coupled via the Lorentz force, which manifests as a source term in the N-S equations. In the case of squeeze-film flows, a magnetic field supplied across the gap (normal to the surfaces) tends to suppress the velocity of a conducting fluid via Joule dissipation. These dissipative MHD forces keep the fluid from being easily squeezed out of the interface, augmenting the role of viscosity and ultimately leading to an increase in load-carrying capacity-or equivalently, damping — of the squeeze-film.

In the general case, the governing modes of physics of MHD flows are intimately coupled. On the one hand, the Maxwell equations explain the coupling of electric and magnetic fields, and on the other hand, the N-S equations explain that of the velocity and pressure fields. Introduction of the Lorentz force into the momentum equation then couples all four fields together, leading to a system that is clearly unmanageable in its general form. As a result, the equations are simplified using dimensional analysis. For example, the magnetic-Reynolds number characterizes the relative effects of induction to magnetic diffusion. We show that in the limit 
of vanishing magnetic-Reynolds number, one-way coupling between the fluid and magnetic field results. ${ }^{10}$

The early studies by $\operatorname{Lin}^{7,11}$ on circular and rectangular MHD squeeze-films were performed for ideally smooth surfaces, permitting an analytical solution to the MHD Reynolds equation via an eigenfunction expansion. However, average roughness measures of the squeeze surfaces are often on the same order of magnitude as the characteristic length of the flow and thus cannot be rightfully neglected. Bujurke and Kudenatti ${ }^{12}$ and Bujurke et al. ${ }^{13}$ solved the MHD squeeze-film problem with a stochastic model of surface roughness. Their work, along with a multitude of others in hydrodynamic lubrication, ${ }^{14-18}$ follows the popular approach of Christensen. ${ }^{19}$ These models employ rather strong assumptions about the nature of the roughness structure in order to capture the statistically expected values of the pressure. Consequently, the resulting pressure fields are always smooth and symmetric, and the local flow perturbations caused by particular surface features are obviously not attainable. This approach has led to several contradicting results from investigators attempting to explain the net effect of isotropic surface roughness on thin-film flows. ${ }^{14-18}$ To avoid such assumptions, we model the problem deterministically using realized surface profiles, which may be generated with various computational methods or acquired from direct profilometry of actual lab samples. With this deterministic approach, the effects of asperity-scale roughness features on both local and global flow properties may be resolved and analyzed. While our model conveniently allows one to prescribe any desired surface topography, we demonstrate a generalized Weierstrass-Mandelbrot fractal $^{8}$ to numerically generate extremely realistic surface profiles.

As mentioned, the MHD Reynolds equation is derived by augmenting the $\mathrm{N}-\mathrm{S}$ equations with the Lorentz force and using the original thin-film assumptions-along with that of small magnetic-Reynolds number-to arrive at the final form of the equation. Because the Lorentz force is defined with a cross product in the classical form, a mathematically consistent reduction of the three-dimensional governing equations into two-dimensions is not possible in the standard vector algebra. Many different flavors of Maxwell's equations and the Lorentz force have been proposed to eliminate the cross product as a means of generalizing the equations to an arbitrary number of dimensions; the most popular of these include the use of differential forms, skew-symmetric tensors, and quaternions. Geometric algebra, ${ }^{20}$ a geometric interpretation of the Clifford algebra, is a rapidly growing framework that naturally encompasses all of these concepts into a unified language. The geometric intuition attached to algebraic structures and operations (e.g., vectors and cross products) in this system enables coordinate-independent computations that are powerful and arguably more intuitive than those of its predecessors. In this work, we implement geometric algebra — and its natural extension: geometric calculus - to formulate the theory without the restrictions imposed by the cross product.

The coefficients of the resulting Poisson problem (i.e., the flow conductivities) are functions of surface topography, viscosity, and MHD properties. In general, discrete representations of real surfaces possess poor regularity, so in order to avoid explicit computation of the gradient of the coefficients, we prefer to work with the weak formulation of the governing equation and implement the Galerkin finite element method (GFEM) to solve the problem. In addition to better performance for non-smooth coefficients, the GFEM naturally allows for many types of domain geometry (e.g., arbitrary polygons or disks), as well as different topologies (e.g., holes and cutouts), to be modeled without any additional effort to the user.

This paper is organized such that Sec. II is a complete and comprehensive development of the underlying physics involved and derivation of the MHD Reynolds equation. Then a discussion on fractal surfaces is provided, in particular, the generalized Weierstrass-Mandelbrot fractal. In Sec. III, we present the weak form of the governing equation and describe the GFEM solution procedure in detail. Finally, the results obtained for a range of numerical test cases are discussed. The examples include perfectly smooth and fractally rough surfaces, imposed magnetic and electric fields, as well as domains with general geometrical and topological features. In the limit of perfectly smooth surfaces, the model produces virtually identical results as the analytical solution. ${ }^{7,11}$ We observe that the load-carrying capacity of the squeeze-film can be significantly improved by increasing the characteristic Hartmann number, as well as generating electric eddy-currents in the fluid. Finally, we employ the model to show that the resulting flow field is significantly influenced by the topography, geometry, and topology of the squeezing boundaries.

\section{FORMULATION OF THE GOVERNING EQUATION}

The governing equation for this model is the MHD Reynolds equation for squeezing flows, which takes into account two classical modes of physics: hydrodynamics and electrodynamics. In this section, we present its complete derivation, starting from the N-S and Maxwell equations. Subsections II A and II B provide context for the general hydrodynamics and electrodynamics, respectively. Then, Subsection II C couples the two governing systems to attain the generalized equation system for MHD flows. A detailed discussion of the relevant MHD physics is given, which includes important dimensionless parameters, Alfvén's theorem, magnetic stresses, and energy dissipation. Finally, Subsection II D applies the thin-film assumptions to the general MHD equations, ultimately leading to the desired MHD Reynolds equation.

\section{A. Hydrodynamics}

The hydrodynamic equations need no modification for compatibility with general MHD other than the addition of a Lorentz body force. Hence, the governing fluid dynamic equations are none other than the classical N-S equations. Over a $n$-dimensional spatial domain $\Omega_{n}$ and time domain $\mathbb{R}^{+}$, the $\mathrm{N}-\mathrm{S}$ equations for incompressible flow are defined such that $\forall(\mathbf{x}, t) \in \Omega_{n} \times \mathbb{R}^{+}$,

$$
\begin{gathered}
\rho\left(\partial_{t} \mathbf{u}+(\mathbf{u} \cdot \nabla) \mathbf{u}\right)+\nabla p-\eta \nabla^{2} \mathbf{u}-\mathbf{f}=\mathbf{0}, \\
\nabla \cdot \mathbf{u}=0,
\end{gathered}
$$


with boundary data on $\Gamma=\Gamma_{D} \cup \Gamma_{N}$ specified by

$$
\begin{gathered}
\mathbf{u}=\mathbf{w} \text { on } \Gamma_{D}, \\
\eta \mathbf{n} \cdot \nabla \mathbf{u}-\mathbf{n} p=\boldsymbol{s} \text { on } \Gamma_{N} .
\end{gathered}
$$

The first and second equations of (1) correspond to the conservation of momentum and mass, respectively. In (1) and (2), $\mathbf{u}: \Omega_{n} \times \mathbb{R}^{+} \rightarrow \mathbb{R}^{n}$ denotes the velocity field, $\rho$ is the fluid density, $p: \Omega_{n} \times \mathbb{R}^{+} \rightarrow \mathbb{R}$ is the scalar pressure field, $\eta$ is the dynamic viscosity of the fluid, and $\mathbf{f}: \Omega_{n} \times \mathbb{R}^{+} \rightarrow \mathbb{R}^{n}$ is a summation of body forces. The pressure acts as a Lagrange multiplier enforcing the divergence-free constraint, and the viscous term quantifies the diffusion of momentum through the flow field. The boundary conditions given in (2) correspond to Dirichlet and Neumann data, w: $\Gamma_{D} \times \mathbb{R}^{n} \rightarrow \mathbb{R}^{n}$ and $\mathbf{s}: \Gamma_{N} \times \mathbb{R}^{+} \rightarrow \mathbb{R}^{n}$, defined on respective parts of the boundary, where $\mathbf{n}$ is the outward-pointing normal vector. In Subsection II C, the Lorentz force will be introduced into $\mathbf{f}$ to provide coupling with the Maxwell equations. The numerical solution of the N-S equations is difficult and computationally expensive; therefore, when possible, problem-specific assumptions are typically made to ease the numerical burden. The Reynolds equation, for example, is a simplified reduction of the N-S equations that lends very well to thin-film problems.

\section{B. Electrodynamics}

We develop the remainder of the theory using the advantageous mathematical structures offered by geometric algebra. While there are numerous advantages of using geometric algebra as opposed to the standard vector algebra, we employ it here predominately to remove the restriction of the cross product (and hence curl) to $\mathbb{R}^{3}$. Our formulation of the governing equation collapses an initially three-dimensional problem involving a cross product into two dimensions; geometric algebra's generalization of the cross product naturally and elegantly handles this dimensional reduction, which would otherwise have been awkward and inconsistent using vector algebra. The relevant ideas and identities needed for this paper are given in the Appendix; however, we refer to the seminal work by Hestenes and Sobczyk ${ }^{20}$ for a thorough discussion on this topic.

Hereafter, we restrict our attention to the important cases where spatial domains are of two and three dimensions, i.e., $n=2,3$. The differential formulation of Maxwell's equations over the geometric algebra $\mathcal{G}\left(\mathbb{R}^{n}\right)$ may be written such that $\forall(\mathbf{x}, t) \in \Omega_{n} \times \mathbb{R}^{+}$,

$$
\begin{gathered}
\nabla \cdot \mathbf{E}=\frac{\rho_{e}}{\varepsilon}, \\
\nabla \wedge\left(\mathbf{B} \mathbf{i}_{n}\right)=0, \\
\nabla \wedge \mathbf{E}=-\partial_{t}\left(\mathbf{B} \mathbf{i}_{n}\right), \\
(\nabla \wedge \mathbf{B}) \mathbf{i}_{n}=-\mu\left(\mathbf{J}+\varepsilon \partial_{t} \mathbf{E}\right),
\end{gathered}
$$

where $\mathbf{E}, \mathbf{J}: \Omega_{n} \times \mathbb{R}^{+} \rightarrow \mathbb{R}^{n}$ are, respectively, the electric and current density fields. The field $\mathbf{B}: \Omega_{n} \times \mathbb{R}^{+} \rightarrow \mathbb{R}^{n(n-1) / 2}$ is the magnetic flux density and referred to as the magnetic field hereafter. Note that $\mathbf{B}$ is defined as a scalar field in two-dimensions and a vector field in three-dimensions. The charge density, magnetic permeability, and electric permittivity of the medium are denoted by $\rho_{e}, \mu$, and $\varepsilon$, respectively. We define the unit pseudoscalars with the Euclidean basis as $\mathbf{i}_{n}=\prod_{j=1}^{n} \mathbf{e}_{j} \in \mathcal{G}\left(\mathbb{R}^{n}\right)$, where the indicated operation between each basis vector is the geometric product (see the Appendix). We note the absence of cross products in (3), which have been replaced by the more general outer (wedge) product. The first and second equations are the electric and magnetic variants of Gauss's law, defining the divergence properties of the electric and magnetic fields. Solenoidal behavior of the fields is given by the last two equations, namely, Faraday's law of induction and the Ampere-Maxwell law. The volumetric form of the electromagnetic force is given as

$$
\mathbf{f}=\rho_{e} \mathbf{E}-(\mathbf{J} \wedge \mathbf{B}) \mathbf{i}_{n} .
$$

Ohm's law closes the system, allowing one to compute the current density via

$$
\mathbf{J}=\sigma\left(\mathbf{E}-(\mathbf{u} \wedge \mathbf{B}) \mathbf{i}_{n}\right) .
$$

The electrical conductivity and velocity field of the conducting medium are denoted by $\sigma$ and $\mathbf{u}$, respectively. In MHD, the conductor is the fluid itself, so its velocity is naturally identical to that of the flow.

In most MHD applications, the Maxwell equations are simplified considerably with the canonical magnetohydrodynamic approximations. ${ }^{21}$ Davidson $^{22}$ showed that for conductors traveling at non-relativistic speeds, $\rho_{e}$ and $\partial_{t} \rho_{e}$ are negligible. This implies that the magnetic part of (4) dominates the electrostatic component, i.e., $\rho_{e}\|\mathbf{E}\| \ll\|\mathbf{J} \wedge \mathbf{B}\|$, and that the displacement current in the Ampere-Maxwell equation is of no significant effect. Conservation of charge then follows

$$
\nabla \cdot \mathbf{J}=0 .
$$

The simplified form of the governing equations for electrodynamics in MHD is then the so-called pre-Maxwellian system,

$$
\begin{gathered}
\nabla \wedge\left(\mathbf{B} \mathbf{i}_{n}\right)=0, \\
\nabla \wedge \mathbf{E}=-\partial_{t}\left(\mathbf{B i} i_{n}\right), \\
(\nabla \wedge \mathbf{B}) \mathbf{i}_{n}=-\mu \mathbf{J},
\end{gathered}
$$

with the Lorentz force

$$
\mathbf{f}=-(\mathbf{J} \wedge \mathbf{B}) \mathbf{i}_{n} .
$$

Ultimately, these approximations have relaxed the Lorentz invariance of the Maxwell equations and produced a similar system that is Galilean invariant. The physical meaning of this is that the pre-Maxwell system excludes the propagation of electromagnetic waves. However since the velocity scales of interest are far from relativistic, this approximation is widely accepted to be well-founded for the majority of MHD applications. We refer the reader to Chap. 2 of Davidson ${ }^{22}$ for a formal and thorough discussion of these assumptions.

\section{Magnetohydrodynamics}

\section{General MHD flow equations}

Coupling of the electromagnetic and fluid dynamic equations yields the governing equation system of general MHD. This is achieved by introducing the volumetric Lorentz force given by (8) into the $\mathrm{N}-\mathrm{S}$ equations,

$$
\rho\left(\partial_{t} \mathbf{u}+(\mathbf{u} \cdot \nabla) \mathbf{u}\right)+\nabla p-\eta \nabla^{2} \mathbf{u}+(\mathbf{J} \wedge \mathbf{B}) \mathbf{i}_{n}=\mathbf{0} .
$$


Ohm, Faraday, and Ampere's laws may be combined to derive a transport equation for $\mathbf{B}$, following

$$
\partial_{t} \mathbf{B}=(\mu \sigma)^{-1} \nabla^{2} \mathbf{B}-\nabla \cdot(\mathbf{u} \wedge \mathbf{B}),
$$

where $(\mu \sigma)^{-1}$ is considered the magnetic diffusivity. This result, commonly referred to as the induction equation, is an advection-diffusion equation for $\mathbf{B}$ and is mathematically analogous to the vorticity equation in fluid mechanics. Consequently, processes that appear in the transport of vorticity of a flow-advection, diffusion, and even vortex stretching - are also present in transport of $\mathbf{B}$; however, the physical reasons that such mechanisms would occur are fundamentally different. Equations (9) and (10), along with constraints $\nabla \cdot \mathbf{u}=\nabla \cdot \mathbf{J}$ $=0$, are the governing equations for generalized, incompressible MHD flow. We note that the second term on the right of (10) is indeed a vector quantity and should not be confused for a scalar; we refer the reader to the Appendix, specifically Table IV, for a precise recipe for computing this term.

\section{Dimensional analysis}

Even with the previously imposed assumptions, the solution of (9) and (10) is a formidable challenge, so we apply appropriate scaling to help reduce complexity. Three important nondimensional parameters characterize the MHD flow, namely, the Reynolds number, the Hartmann number, and the magnetic-Reynolds number. The Reynolds number is, as usual, the measure of inertial to viscous forces. The Hartmann number, defined with a characteristic length scale $l$ as

$$
M=\|\mathbf{B}\| l \sqrt{\frac{\sigma}{\eta}},
$$

is a relative measure of the Lorentz force to the viscous force. Finally, the magnetic-Reynolds number weighs the strength of advection against diffusion in the induction equation (10) and is defined as

$$
R_{m}=\|\mathbf{u}\| \mu \sigma l .
$$

This parameter largely determines the behavior of the MHD flow as it relates to Alfvén's theorem, which is a magnetic field analog of Helmholtz's first law and Kelvin's theorem for inviscid fluids. Alfvén's theorem ${ }^{23}$ describes how the magnetic field lines are "frozen" into a perfectly conducting fluid in the same way that a vortex filament is frozen into an ideal fluid. This may be seen by integrating Faraday's law with Stokes' theorem to reveal that in the limit $\sigma \rightarrow \infty$ (i.e., an ideal conductor), there is no change in the magnetic flux through any manifold bounded by a closed loop made of material fluid parcels. This suggests that the field lines are always attached to the same fluid parcel and perfectly advected with the flow, which preserves the topology of the magnetic field. As the field lines are stretched, a tension is induced applying a back-reaction on the flow via the Lorentz force. We again refer to Davidson ${ }^{22}$ for a detailed discussion on the matter of high- $R_{m}$ flows.

Squeeze-films are typically characterized by small length and velocity scales; therefore, we enforce a small- $R_{m}$ assumption for this work. In this case where $R_{m} \ll 1$, the induced electric current does not create a secondary magnetic field strong enough to significantly alter the original field. As a result, the field lines are not advected with the flow. The implications of this may be elucidated upon further investigation of $R_{m}$. This parameter manifests itself in the dimensionless form of the induction equation, which we demonstrate first by nondimensionalizing Ampere's law with the following scales:

$$
\nabla=l^{-1} \tilde{\nabla}, \quad \mathbf{B}=\|\mathbf{B}\| \tilde{\mathbf{B}}, \text { and } \quad \mathbf{J}=-\sigma\|\mathbf{u}\|\|\mathbf{B}\| \tilde{\mathbf{J}} .
$$

The tildes indicate dimensionless quantities. After a minimal amount of algebraic manipulation, a dimensionless Ampere's law may then be written as

$$
(\|\mathbf{u}\| \mu \sigma l) \tilde{\mathbf{J}}=(\tilde{\nabla} \wedge \tilde{\mathbf{B}}) \mathbf{i}_{n},
$$

where the scaling factor on the left is identical to $R_{m}$ defined in (12). According to (13) when $R_{m} \ll 1$, a small perturbation of the magnetic field can induce a current of significant magnitude - from the other perspective, a moderately sized current may only lead to small disturbances of the magnetic field. Therefore in low- $R_{m}$ scenarios, an imposed field may greatly influence the flow despite the flow's inability to significantly alter the magnetic field; i.e., one-way coupling is assumed.

Extending this dimensional analysis to the induction equation (10), along with the time scale $t=\left(l\|\mathbf{u}\|^{-1}\right) \tilde{t}$, a dimensionless induction equation may be formulated as

$$
R_{m}\left(\partial_{\tilde{t}} \tilde{\mathbf{B}}+\tilde{\nabla} \cdot(\tilde{\mathbf{u}} \wedge \tilde{\mathbf{B}})\right)=\tilde{\nabla}^{2} \tilde{\mathbf{B}} .
$$

Consequently taking $R_{m} \rightarrow 0$, the induction equation, which is generally an unsteady transport equation coupled to the fluid mechanics equations through the advective term, reduces to an uncoupled, steady-state diffusion equation,

$$
\tilde{\nabla}^{2} \tilde{\mathbf{B}}=\mathbf{0} .
$$

It is now evident that any uniform initial $\mathbf{B}_{0}$ satisfies this equation, implying that $\mathbf{B}=\mathbf{B}_{0}$ holds at all times. This is referred to as the quasi-steady assumption of the magnetic field since, in reality, some perturbation of the field lines is required to induce the Lorentz force. Ultimately, the low- $R_{m}$ assumption has reduced the challenge of simultaneously solving (9) and (10) to solving just (9) (with the divergence-free constraint) for determination of the flow.

\section{Magnetic damping}

Our application is particularly concerned with the suppression of the flow via magnetic damping, which is revealed upon further inspection of the Lorentz force. Let us briefly restrict our attention to $\mathbb{R}^{3}$ so that we may replace the outer product with a cross product according to the dual relation described in the Appendix, $-(\mathbf{J} \wedge \mathbf{B}) \mathbf{i}_{3}=\mathbf{J} \times \mathbf{B}$. The Lorentz force may be viewed as the divergence of the so-called Maxwell stress tensor, written as

$$
\mathbf{J} \times \mathbf{B}=\nabla \cdot \boldsymbol{\pi},
$$

where $\boldsymbol{\pi}=\mathbf{B} \otimes \mathbf{B} / \mu-\left(\|\mathbf{B}\|^{2} / 2 \mu\right) \mathbf{I}$ is the magnetic stress tensor decomposed here into deviatoric and volumetric parts. The tension in a field line is obtained by computation of the principal stress states, which correspond to the eigenpairs of the deviatoric part of $\boldsymbol{\pi}$. It is simple to show that the deviator tensor has one nonzero eigenvalue $\lambda=\mu^{-1}\|\mathbf{B}\|^{2}$ corresponding to eigenvector $\mathbf{v}=\|\mathbf{B}\|^{-1} \mathbf{B}$, i.e., the unit tangent vector to field. Thus, the stress state developed in the $\mathbf{B}$-field is completely 


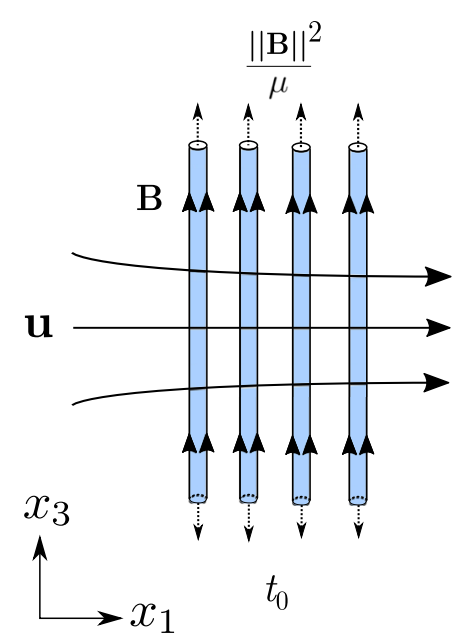

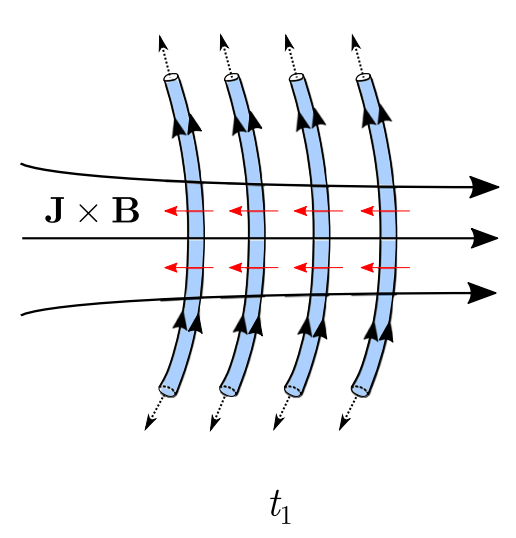

FIG. 1. Magnetic field lines deforming with the flow stream. The Maxwell tensions $\mu^{-1}\|\mathbf{B}\|^{2}$ develop a net horizontal component at $t=t_{1}$, leading to a Lorentz force $\mathbf{J} \times \mathbf{B}$ that does work against the flow. characterized as a tension of magnitude $\mu^{-1}\|\mathbf{B}\|^{2}$. This process is visually shown in Fig. 1, where an initially vertical magnetic field at $t=t_{0}$ has been slightly perturbed by the flow at $t=t_{1}$. The deformation of the magnetic field causes the once-vertical tension vectors to rotate such that they develop a net component directed against the flow velocity. The horizontal tension component is equilibrated by an electric current in the $-x_{2}$ direction (out of the page), where the Lorentz force then acts in the $-x_{1}$ direction to suppress the flow velocity at $t=t_{2}$.

Remark 1. Magnetic dissipation is anisotropic, unlike that of isotropic, Newtonian viscosity. The action only opposes the fluid motion normal to the magnetic field lines, and the strength of the action is dependent on $R_{m}$.

\section{Magnetohydrodynamic Reynolds equation for squeeze-film flows}

We now derive the MHD Reynolds equation and begin by making the following assumptions: (1) the fluid is inertialess, Newtonian, and thin (i.e., $\operatorname{Re} \ll 1,\left\|\partial_{11} \mathbf{u}\right\|,\left\|\partial_{22} \mathbf{u}\right\|$ $\ll\left\|\partial_{33} \mathbf{u}\right\|$, and $\partial_{3} p=0$ ), (2) the Lorentz force is the only body force, and (3) the magnetic film is quasi-steady. The first and second of these assumptions are canonical in terms of thinfilm flows. These were among the ad hoc assumptions made by Reynolds; ${ }^{5}$ however, they have since been redeveloped in a more rigorous and convincing manner by Elrod ${ }^{24}$ and Langlois. ${ }^{25}$ Such assumptions are widely still used in thin-film analyses; however, there is considerable work that is also being performed in retaining the inertia terms for appropriate cases. We plan to discuss our model's capabilities regarding this point in a future paper. We note that the implications of the third assumption have already been discussed in Sec. II C.

With the classical thin-film assumptions in place, (9) is reduced, along with the divergence-free constraint, to

$$
\begin{gathered}
\nabla p=\eta \partial_{33} \mathbf{u}-\mathbf{i}_{3} \mathbf{J} \wedge \mathbf{B}, \\
\nabla \cdot \mathbf{u}=0 .
\end{gathered}
$$

The Lorentz force may be expanded with Ohm's law to

$$
-\mathbf{i}_{3} \mathbf{J} \wedge \mathbf{B}=-\sigma\left(\mathbf{i}_{3} \mathbf{E} \wedge \mathbf{B}+(\mathbf{u} \wedge \mathbf{B}) \cdot \mathbf{B}\right),
$$

where the second term on the right implies that mutually parallel $\mathbf{u}$ and $\mathbf{B}$ components do not contribute to the Lorentz force. Considering (18) and the schematic in Fig. 2, (17) is expanded into its component form,

$$
\begin{gathered}
\partial_{1} p=\eta \partial_{33} u_{1}+\sigma\left(E_{2}\left\|\mathbf{B}_{0}\right\|-\left\|\mathbf{B}_{0}\right\|^{2} u_{1}\right), \\
\partial_{2} p=\eta \partial_{33} u_{2}-\sigma\left(E_{1}\left\|\mathbf{B}_{0}\right\|+\left\|\mathbf{B}_{0}\right\|^{2} u_{2}\right), \\
\partial_{3} p=\eta \partial_{33} u_{3}=0,
\end{gathered}
$$

where $E_{1}$ and $E_{2}$ are the transverse components of the supplied electric field. No-slip boundary conditions are applied to the surfaces of the plate, following

$$
\mathbf{u}\left(x_{1}, x_{2}, 0\right)=\mathbf{0}, \quad \mathbf{u}\left(x_{1}, x_{2}, h\right)=\left[\begin{array}{lll}
0 & 0 & \partial_{t} h
\end{array}\right]^{T},
$$

where $h$ is the instantaneous film thickness. In the entirety of this work, we assume the squeezing boundaries to be

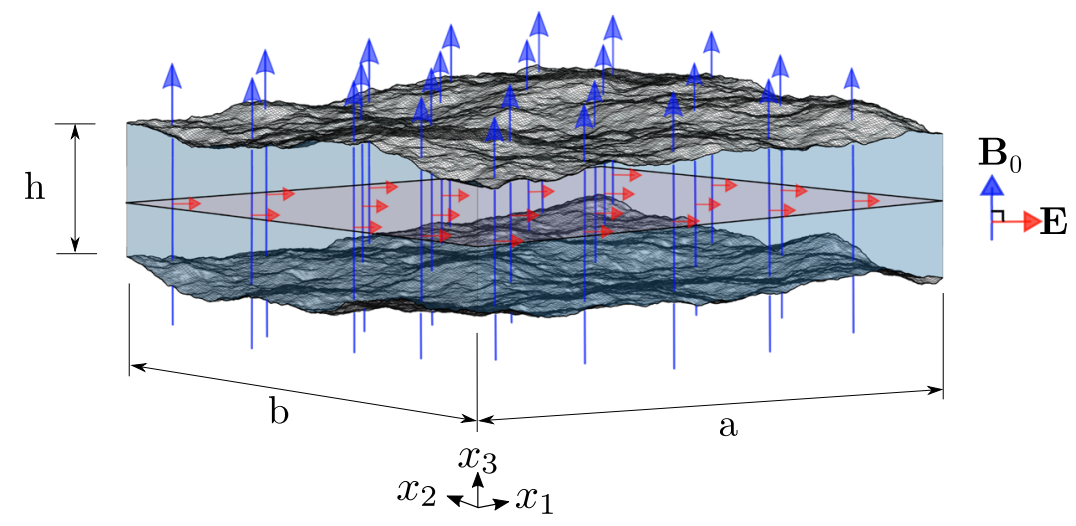

FIG. 2. Configuration of a rough squeeze-film with an imposed uniform, vertical magnetic field. The depth is changing at a rate $\partial_{t} h$. Only the electric field components perpendicular to the magnetic field will influence the flow. 
perfectly insulated from the conducting fluid. This ensures that the induced electric current remains solenoidal within the fluid rather than flowing into the bounding surfaces. Without loss of generality, we have taken the entire bottom surface to be the $x_{3}=0$ datum, implying that the top surface is located at $x_{3}=h\left(x_{1}, x_{2}\right)$. Expressions for the velocity components are obtained by integrating (19) subject to (20). Substituting the Hartmann number with the initial film-depth $h_{0}$ as the characteristic length scale, the following velocity expressions are attained:

$$
\begin{aligned}
u_{1}= & \frac{h_{0}^{2}}{\eta M^{2}}\left(\partial_{1} p-\frac{M \sqrt{\eta \sigma}}{h_{0}} E_{2}\right)\left[\cosh \left(M x_{3} / h_{0}\right)-1\right. \\
& \left.-\left(\cosh \left(M h / h_{0}\right)-1\right)\left(\frac{\sinh \left(M x_{3} / h_{0}\right)}{\sinh \left(M h / h_{0}\right)}\right)\right], \\
u_{2}= & \frac{h_{0}^{2}}{\eta M^{2}}\left(\partial_{2} p+\frac{M \sqrt{\eta \sigma}}{h_{0}} E_{1}\right)\left[\cosh \left(M x_{3} / h_{0}\right)-1\right. \\
& \left.-\left(\cosh \left(M h / h_{0}\right)-1\right)\left(\frac{\sinh \left(M x_{3} / h_{0}\right)}{\sinh \left(M h / h_{0}\right)}\right)\right], \\
u_{3}= & \frac{x_{3}}{h} \partial_{t} h .
\end{aligned}
$$

The resulting velocity profiles are characteristic of Hartmann flow, which feature a flattened Poiseuille-type profile. The magnitude of flattening depends on the Hartmann number and is caused by the Lorentz force tending to suppress the bulk flow more than the boundary layers, which in the case of Hartmann flows are termed Hartmann layers. We have mentioned that the boundaries in our work are considered insulated; however, the Hartmann effect is greatly amplified in scenarios with electrically conducting boundaries. This ultimately would lead to significant increases in damping-we refer the interested reader to the work of Müller and Bühler ${ }^{26}$ for more information regarding conducting boundaries of Hartmann flows.

The velocity components are then eliminated for pressure by first integrating the incompressibility constraint over the film thickness. Then applying Leibniz's rule to bring the integration inside the divergence operator, we get

$$
\nabla \cdot \int_{0}^{h\left(x_{1}, x_{2}, t\right)} \mathbf{u} d x_{3}=0
$$

which implies that continuity is now enforced in a depthaveraged sense. Finally, the MHD Reynolds equation is obtained by combining (21) and (22), subject to the boundary data in (20), and computing the integrations,

$$
\frac{h_{0}^{3}}{\eta M^{3}} \nabla \cdot\left[\left(\frac{M h}{h_{0}}-2 \tanh \left(M h / 2 h_{0}\right)\right)\left(\nabla p-\frac{M \sqrt{\eta \sigma}}{h_{0}} \mathbf{E i}_{2}\right)\right]=\partial_{t} h .
$$

Note that (23) is now a two-dimensional problem due to the integration over the $x_{3}$-dimension. The (pseudo-)vector $\mathbf{E i}_{2}=\left[\begin{array}{ll}-E_{2} & E_{1}\end{array}\right]^{T}$ is the Hodge dual of $\mathbf{E} \in \mathbb{R}^{2}$, which in traditional vector algebra is equivalent to the orthogonal complement of $\mathbf{E} \in \mathbb{R}^{3}$ projected onto the $\mathbf{e}_{12}$ plane.

The non-dimensional form of (23) is obtained with the following dimensionless parameters:

$$
\begin{gathered}
\tilde{x}_{1}=\frac{x_{1}}{a}, \quad \tilde{x}_{2}=\frac{x_{2}}{b}, \quad \tilde{h}=\frac{h}{h_{0}}, \\
\tilde{p}=-\frac{p h_{0}^{3}}{\eta a^{2} \partial_{t} h}, \quad \tilde{\mathbf{E}}=-\frac{\mathbf{E} h_{0}}{a \partial_{t} h\left\|\mathbf{B}_{0}\right\|}, \quad \beta=\frac{a}{b},
\end{gathered}
$$

where $\beta$ is commonly known as the aspect ratio. Furthermore, defining the flow conductivity as

$$
\kappa(\tilde{\mathbf{x}})=M \tilde{h}(\tilde{\mathbf{x}})-2 \tanh (M \tilde{h}(\tilde{\mathbf{x}}) / 2),
$$

we arrive at the dimensionless form of the MHD Reynolds equation with homogeneous Dirichlet boundary data,

$$
\begin{gathered}
\tilde{\nabla} \cdot(\kappa \tilde{\nabla} \tilde{p})=-M^{3}\left(1+M^{-1} \mathbf{i}_{2} \tilde{\nabla} \wedge(\kappa \tilde{\mathbf{E}})\right), \quad \tilde{\mathbf{x}} \in \mathrm{P}\left(\Omega_{3}\right)=\Omega_{2}, \\
\tilde{p}=0, \quad \tilde{\mathbf{x}} \in \mathrm{P}\left(\Gamma_{2}\right)=\Gamma_{1} .
\end{gathered}
$$

The three-dimensional domain $\Omega_{3}$ and its two-dimensional boundary $\Gamma_{2}$ are collapsed to their respective two- and onedimensional counterparts using the trivial projection operation P: $\left(\tilde{x}_{1}, \tilde{x}_{2}, \tilde{x}_{3}\right) \mapsto\left(\tilde{x}_{1}, \tilde{x}_{2}\right)$, which simply discards the $x_{3}-$ component of the vector argument. Finally, the dimensionless gradient operator is defined as

$$
\tilde{\nabla}=\left(\begin{array}{c}
\tilde{\partial}_{1} \\
\beta \tilde{\partial}_{2}
\end{array}\right)
$$

The performance of the squeeze-film is, in general, characterized by the so-called load-carrying capacity $\tilde{W}$. This is easily attained by integrating the resulting pressure solution of (25) over the two-dimensional domain; hence, the load carrying capacity is defined as

$$
\tilde{W}=\int_{\Omega_{2}} \tilde{p} d \Omega_{2}
$$

In previous studies on MHD squeeze-films, an imposed electric field has been neglected-doing so results in (25) without the second term on the right hand side. This term is equivalent to the "scalar curl" of $\kappa \tilde{\mathbf{E}}$, which is not consistently defined in standard vector calculus but is handled naturally in geometric calculus. We may attain a better understanding of this term by expanding with the product rule,

$$
-\mathbf{i}_{2} M^{2} \tilde{\nabla} \wedge(\kappa \tilde{\mathbf{E}})=-\mathbf{i}_{2} M^{2}(\kappa(\tilde{\nabla} \wedge \tilde{\mathbf{E}})+(\tilde{\nabla} \kappa) \wedge \tilde{\mathbf{E}}) .
$$

Evidently for constant $\kappa$, the last term on the right vanishes, implying that a conservative (i.e., irrotational) E-field will not influence $\tilde{W}$. This fact will be demonstrated in the numerical examples. However for rough surfaces, $\kappa$ is not constant, and conservative E-fields may well alter the load-carrying capacity. This mechanism is intuitive and explained as follows. Assuming $M$ does not vary across the domain, $\kappa$ is directly associated with the film-depth following (24), so for any point $\tilde{\mathbf{x}} \in \Omega_{2}$, the component of $\tilde{\mathbf{E}}$ perpendicular to $\tilde{\nabla} \kappa$ creates a force that, depending on its orientation, pushes the fluid toward a deeper or shallower depth. For example, when $\left.(\tilde{\nabla} \kappa) \wedge \tilde{\mathbf{E}}\right|_{\tilde{\mathbf{x}}}>0$, the flow is coerced toward increasing $\tilde{h}$, effectively leading to a rise in flow conductivity and a differential decrease in $\tilde{W}$. In the opposite case where $\left.(\tilde{\nabla} \kappa) \wedge \tilde{\mathbf{E}}\right|_{\tilde{\mathbf{x}}}<0$, the resulting force pushes the flow toward decreasing film-thickness, effectively lowering the flow conductivity and differentially increasing $\tilde{W}$.

Remark 2. It is readily shown that (25) reduces to the standard, purely hydrodynamic Reynolds equation as $M \rightarrow 0$, implying that the current MHD formulation is consistent. 


\section{SURFACE CHARACTERIZATION}

The confining surfaces of squeeze-film flows typically have roughness features on the same order as the mean filmdepth. For this reason, the surface asperities are likely to significantly influence the flow. ${ }^{12}$ From a stochastic viewpoint, surface roughness is often assumed to be a stationary random process ${ }^{27}$ however, investigations by Sayles and Thomas ${ }^{28}$ show that, in many cases, the contrary is true. Furthermore, the power spectra of many engineering surfaces follow inverse power laws over a large range of wavelengths, suggesting that statistically similar features reappear over every length scale in this range. ${ }^{29}$ As a result, roughness measurements for the same surface may not be unique, in general, as such measurements depend on the resolution of the measuring device itself. This behavior is associated with geometric selfsimilarity and self-affinity, which are intrinsic properties of fractals.

Surface topography is integrated into the proposed model by specifying the function $\tilde{h}(\tilde{\mathbf{x}})$ that results as the difference of the upper and lower surface elevations. Any method of surface generation or direct measurement may be used to construct $\tilde{h}$; however, we implement a generalized Weierstrass-Mandelbrot fractal function to digitally produce realistic surfaces that possess the self-affine properties previously mentioned.

A fractal may be defined as a set whose Hausdorff dimension, $D_{H}$, exceeds its topological dimension. The topological dimension of a set, $D_{T} \in \mathbb{N}_{0}^{+}$, closely follows the popular understanding of dimension, where it only makes sense as a positive, natural number. On the other hand, the Hausdorff dimension generalizes this concept based on measure theory. In particular, the Hausdorff dimension is based on the Hausdorff measure; e.g., see the work of Badii and Politi. ${ }^{30}$ It follows from this definition of dimension that $D_{H}$ no longer needs to be a natural number and can be fractional. Although the notion of fractional dimension may be bothersome, the Hausdorff dimension (referred to hereafter as the fractal dimension) is a useful mathematical construction and is paramount to the study of post-Euclidean geometry, which features fractal entities.

Ausloos and Berman ${ }^{8}$ generalized the famous onedimensional W-M function ${ }^{31}$ to multiple spatial dimensions. In Cartesian coordinates, we present the non-dimensional form of the generalized W-M function for isotropic surfaces ${ }^{32}$ as

$$
\begin{aligned}
\tilde{\zeta}\left(\tilde{x}_{1}, \tilde{x}_{2}\right)= & \tilde{L}\left(\frac{\tilde{G}}{\tilde{L}}\right)^{\left(D_{H}-2\right)}\left(\frac{\ln \gamma}{M_{0}}\right)^{1 / 2} \sum_{m=1}^{m_{\max }} \sum_{n=0}^{n_{\max }} \gamma^{\left(D_{H}-3\right) n} \\
& \times\left[\cos \phi_{m, n}-\cos \left(\frac{2 \pi \gamma^{n}\left[\left(\tilde{x}_{1}\right)^{2}+\beta^{2}\left(\tilde{x}_{2}\right)^{2}\right]^{1 / 2}}{\tilde{L}}\right.\right. \\
& \left.\left.\times \cos \left(\theta-\alpha_{m}\right)+\phi_{m, n}\right)\right],
\end{aligned}
$$

where $\tilde{G}$ is a frequency-invariant length scale termed the fractal roughness, $m_{\max }$ is a finite number of azimuthal directions in which surface corrugations are propagated, and $n_{\max }$ corresponds to a spatial cut-off frequency computed by

$$
n_{\max }=\operatorname{int}\left[\frac{\log \left(\tilde{L} / \tilde{L}_{s}\right)}{\log \gamma}\right],
$$

where the int[.] operator denotes the integer value of the argument. The angles $\theta=\tan ^{-1}\left(\tilde{x}_{2} / \tilde{x}_{1}\right)$ and $\alpha_{m}=\pi m / m_{\max }$ together describe the wave directions. The length scales $\tilde{L}$ and $\tilde{L}_{s}$ denote the sample length of the surface and the minimum wavelength, respectively. The frequencies $\gamma^{n}$ progress geometrically, and the sequence of phases $\phi_{m, n}$ may be chosen randomly or deterministically. The scale-invariant fractal parameters $\tilde{G}$ and $D_{H}$ now uniquely characterize the surface roughness. A fractal representation of a surface with topological dimension $D_{T}=2$ will have a corresponding fractal dimension $D_{H} \in \mathbb{R}[2,3)$. For isotropic roughness, the fractal dimension $D_{H}$ may be estimated by the log-log slope of the surface's one-dimensional power spectrum, which is approximated according to Majumdar and Tien ${ }^{29}$ by

$$
\tilde{S}(\tilde{k})=\frac{\tilde{G}^{2\left(D_{H}-1\right)}}{2 \ln \gamma} \frac{1}{\tilde{k}^{\left(5-2 D_{H}\right)}},
$$

where $\tilde{k}$ is the spatial frequency (i.e., wavenumber) of the surface. The fractal roughness $\tilde{G}$ is then determined such that Parseval's theorem holds,

$\tilde{\sigma}_{\zeta}^{2}=\int_{\tilde{k}_{l}}^{\tilde{k}_{h}} \tilde{S}(\tilde{k}) d \tilde{k}=\frac{\tilde{G}^{2\left(D_{H}-1\right)}}{2 \ln \gamma} \frac{1}{4-2 D_{H}}\left(\frac{1}{\tilde{k}_{l}^{4-2 D_{H}}}-\frac{1}{\tilde{k}_{h}^{4-2 D_{H}}}\right)$,

where $\sigma_{\zeta}^{2}$ is the variance of the profile, while $\tilde{k}_{l}$ and $\tilde{k}_{h}$ are the lower and upper cut-off frequencies, respectively.

As a visual example displaying the role of fractal dimension, two surfaces that only differ in $D_{H}$ are generated and shown in Figs. 3(a) and 3(b). Surfaces (a) and (b) are the

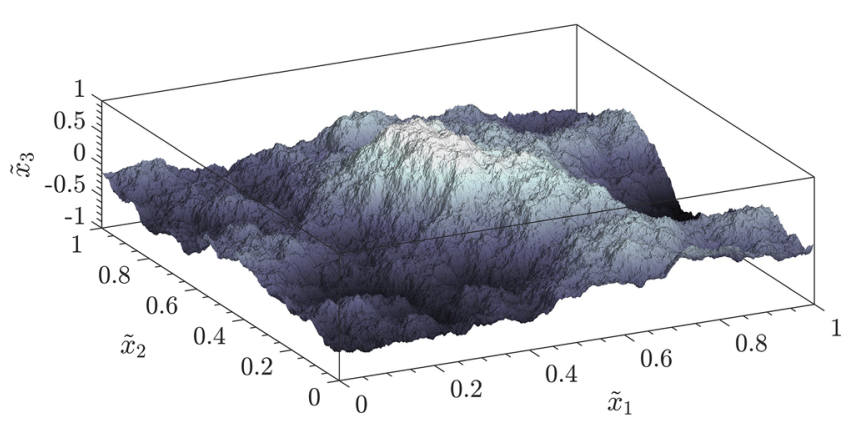

(a)

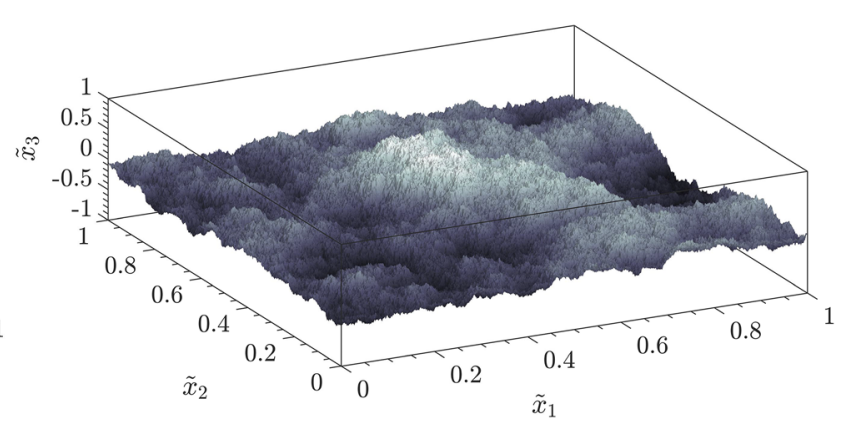

(b)

FIG. 3. Surfaces generated using the generalized Weierstrass-Mandelbrot fractal function with $\tilde{\sigma}_{\zeta}^{2}=0.15$. Surface (a) has fractal dimension $D_{H}=2.2$, and surface (b) has fractal dimension $D_{H}=2.5$. 
results of (28) with fractal dimensions $D_{H}=2.2$ and $D_{H}=2.5$, respectively. Comparison of the plots illustrates how the larger fractal dimension in (b) corresponds to a surface possessing a greater amplitude-ratio between high-low frequency waves compared to that of (a). In other words, the power spectrum of (b) is shifted to the right of (a), resulting in larger amplitudes for the higher-frequencies.

\section{THE FINITE ELEMENT METHOD}

The Galerkin finite element method (GFEM) is implemented to solve the MHD Reynolds equation (25). For notational ease, we will denote the two-dimensional domain $\Omega_{2}=\Omega$ and its boundary $\Gamma_{1}=\Gamma$. The point of departure for finite element methods is the weak formulation. Assuming a suitable solution space $\mathcal{S}$ and test space $\mathcal{V}$, the weak form of (25) follows: find $\tilde{p} \in \mathcal{S}$ such that $\forall v \in \mathcal{V}$,

$$
\begin{aligned}
\int_{\Omega} \kappa \tilde{\nabla} \tilde{p} \cdot \tilde{\nabla} v d \Omega= & \int_{\Gamma}\left(\kappa v \tilde{\nabla} \tilde{p} \cdot \mathbf{n}+\kappa v M \mathbf{i}_{2}(\tilde{\mathbf{E}} \wedge \mathbf{n})\right) d \Gamma \\
& +\int_{\Omega} M^{3}\left(v+\kappa M^{-1}\left(\mathbf{i}_{2} \tilde{\mathbf{E}}\right) \cdot \tilde{\nabla} v\right) d \Omega
\end{aligned}
$$

The solution and test spaces are defined, respectively, as

$$
\begin{aligned}
& \mathcal{S}=\left\{\tilde{p}(\tilde{\mathbf{x}}) \in \mathcal{H}^{1}(\Omega): \tilde{p}(\tilde{\mathbf{x}})=g_{D} \quad \forall \tilde{\mathbf{x}} \in \Gamma_{D}\right\}, \\
& \mathcal{V}=\left\{v(\tilde{\mathbf{x}}) \in \mathcal{H}^{1}(\Omega): v(\tilde{\mathbf{x}})=0 \quad \forall \tilde{\mathbf{x}} \in \Gamma_{D}\right\},
\end{aligned}
$$

where $\mathcal{H}^{1}(\Omega)$ is the Sobolev space with square-integrable first derivatives. Noting the boundary data specified by (25), we see that $g_{D}=0$ on all portions of the boundary so that $\mathcal{S}=\mathcal{V}$ for the homogeneous case. Also because $\Gamma_{N}=\emptyset$, the surface integral term vanishes from (31). Note that the gradient of $\kappa$ does not need to be computed explicitly as it would in the strong formulation; consequently, the $\mathcal{H}^{1}$ regularity requirement of $\kappa$ is relaxed to $L^{2}$, which is desirable since realistic surfaces often possess poor smoothness properties. The lefthand integral of (31) may be written as a multilinear form a $(\tilde{p}, v ; \kappa): \mathcal{S} \times \mathcal{V} \times L^{2}(\Omega) \rightarrow \mathbb{R}$, while the right-hand integral is a form $1(v ; \kappa, \tilde{\mathbf{E}}): \mathcal{V} \times L^{2}(\Omega) \times\left[L^{2}(\Omega)\right]^{2} \rightarrow \mathbb{R}$ linear only in its first argument. The weak problem in a more compact form then follows: find $\tilde{p} \in \mathcal{S}$ such that $\forall v \in \mathcal{V}$,

$$
\mathrm{a}(\tilde{p}, v ; \kappa)=1(v ; \kappa, \tilde{\mathbf{E}}) .
$$

The computational domain $\Omega$ is discretized by an admissible triangular mesh $\mathcal{T}^{h}$, where each element $\omega \in \mathcal{T}^{h}$ is the image of a bijective, affine mapping $T_{\omega}: \hat{\omega} \rightarrow \omega$ from a reference triangle $\hat{\omega}$, which may be defined as

$$
T_{\omega}(\hat{\mathbf{x}})=\mathbf{D} \hat{\mathbf{x}}+\tilde{\mathbf{x}}_{1},
$$

where $\mathbf{D} \in \mathbb{R}^{2 \times 2}$ is the Jacobian matrix with columns $\mathbf{D}_{(1)}$ $=\tilde{\mathbf{x}}_{2}-\tilde{\mathbf{x}}_{1}$ and $\mathbf{D}_{(2)}=\tilde{\mathbf{x}}_{3}-\tilde{\mathbf{x}}_{1}$. The vectors $\tilde{\mathbf{x}}_{1}, \tilde{\mathbf{x}}_{2}$, and $\tilde{\mathbf{x}}_{3}$ are the global coordinates of the corresponding element nodes. We define a finite dimensional space $\mathcal{U}^{h}$ associated with $\mathcal{T}^{h}$ as

$$
\mathcal{U}^{h}=\left\{v^{h} \in \mathcal{C}(\bar{\Omega}):\left.v^{h}\right|_{\omega} \circ T_{\omega} \in \mathcal{P}^{1}(\hat{\omega}) \forall \omega \in \mathcal{T}^{h}\right\},
$$

with $\mathcal{C}$ denoting the space of continuous functions over the closure $\bar{\Omega}$, while $\mathcal{P}^{1}(\hat{\omega})$ denotes the space of first-order polynomials over the reference element. We then introduce conforming finite element subspaces of the infinite dimensional trial and test spaces,

$$
\mathcal{S}^{h}=\mathcal{S} \cap \mathcal{U}^{h} \subset \mathcal{S}, \quad \mathcal{V}^{h}=\mathcal{V} \cap \mathcal{U}^{h} \subset \mathcal{V} .
$$

The first order Lagrange polynomials, with element nodes as the interpolation points, form a complete basis for these finite-dimensional spaces. Each node has a corresponding global basis function $\Psi_{i}(\tilde{\mathbf{x}})$ that is compactly supported over all elements that share the node.

Rather than performing computations globally, we take advantage of the compactness of the basis functions and perform all integrations over $\hat{\omega}$. Local basis functions $\hat{\Psi}_{i}$ are introduced for each node of $\hat{\omega}$ and defined such that they are the pullback of $\Psi_{i}$ by $T_{\omega}$,

$$
\hat{\Psi}_{i}=\Psi_{i} \circ T_{\omega}
$$

Using the local basis functions, we construct a projection of the solution onto $\mathcal{S}^{h}$ by

$$
\left.\tilde{p}^{h}\right|_{\omega}=\sum_{j=1}^{3} \tilde{p}_{j} \hat{\Psi}_{j},
$$

where $\tilde{p}_{j}$ are the nodal degrees of freedom. In the Galerkin formulation, the test functions are chosen to be identical to the basis functions. The scalar field $\kappa$ and vector field $\tilde{\mathbf{E}}$ may also be interpolated over the elements with the same basis functions. The integrals in (31) are cast over the reference element; then, using substitution and the chain rule, we attain the element-level discrete bilinear and linear forms,

$$
\begin{aligned}
\mathrm{a}_{\omega}^{h}\left(\hat{\Psi}_{j}, \hat{\Psi}_{i} ; \kappa\right)= & \int_{\hat{\omega}}\left(\sum_{k=1}^{3} \kappa_{k} \hat{\Psi}_{k}\right)\left(\left(\hat{\nabla} \hat{\Psi}_{j}\right)^{T} \mathbf{G} \hat{\nabla} \hat{\Psi}_{i}\right)|\mathbf{D}| d \hat{\omega}, \\
\mathbf{l}_{\omega}^{h}\left(\hat{\Psi}_{i} ; \kappa, \tilde{\mathbf{E}}\right)= & \int_{\hat{\omega}} M^{3} \hat{\Psi}_{i}+M^{2}\left(\sum_{k=1}^{3} \kappa_{k} \hat{\Psi}_{k}\right) \\
& \times\left(\sum_{k=1}^{3} \mathbf{i}_{2} \tilde{\mathbf{E}}_{k} \hat{\Psi}_{k}\right)^{T}\left(\mathbf{D}^{-T} \hat{\nabla} \hat{\Psi}_{i}\right)|\mathbf{D}| d \hat{\omega},
\end{aligned}
$$

where $\mathbf{G}=\left(\mathbf{D}^{T} \mathbf{D}\right)^{-1}$ is the element metric tensor, $\hat{\nabla}$ is the dimensionless local gradient operator, and $|\mathbf{D}|$ is the Jacobian determinant. The corresponding elemental stiffness matrices $\left.\mathbf{K}\right|_{\omega}$ and load vectors $\left.\mathbf{F}\right|_{\omega}$ are computed following

$$
\begin{aligned}
\left.K_{i j}\right|_{\omega} & =\mathrm{a}_{\omega}^{h}\left(\hat{\Psi}_{j}, \hat{\Psi}_{i} ; \kappa\right), \\
\left.F_{i}\right|_{\omega} & =I_{\omega}^{h}\left(\hat{\Psi}_{i} ; \kappa, \tilde{\mathbf{E}}\right)
\end{aligned}
$$

for $i, j=1,2,3$, where the integrations are performed numerically using a 4-point Gauss quadrature. The elemental matrices and vectors are assembled into the global algebraic system,

$$
\mathbf{K} \tilde{\mathbf{p}}=\mathbf{F},
$$

such that the global stiffness matrix $\mathbf{K} \in \mathbb{R}^{N_{\text {in }} \times N_{\text {in }}}$ and load vector $\mathbf{L} \in \mathbb{R}^{N_{\text {in }}}$ follow

$$
\begin{aligned}
\mathbf{K} & =\left.\underset{e=1}{\mathbf{A}} \mathbf{K}\right|_{\omega_{e}} \\
\mathbf{L} & =\left.\underset{e=1}{\mathbf{A}} \mathbf{L}\right|_{\omega_{e}},
\end{aligned}
$$

where $\mathbf{A}$ is the finite element assembly operator over the total number of elements $N_{e}$ and $\tilde{\mathbf{p}} \in \mathbb{R}^{N_{\text {in }}}$ is the solution vector corresponding to the $N_{\text {in }}$ interior nodes. 


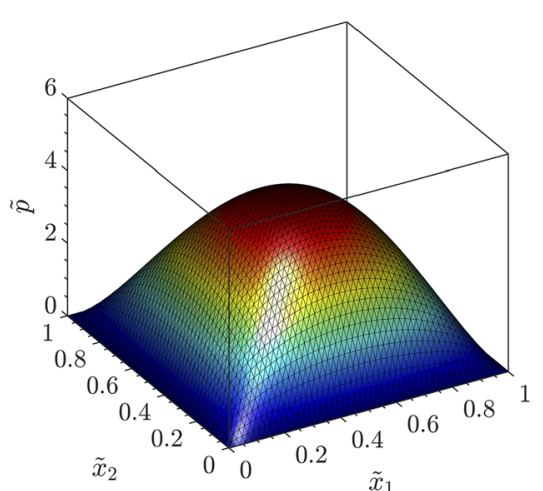

(a)

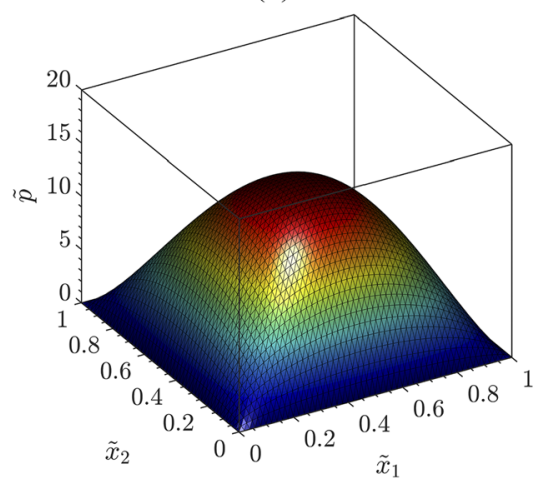

(c)

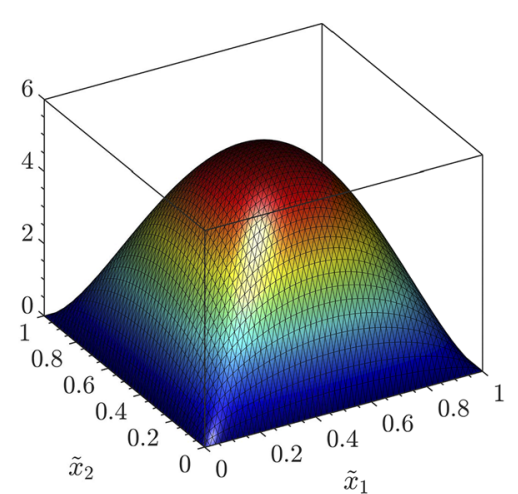

(b)

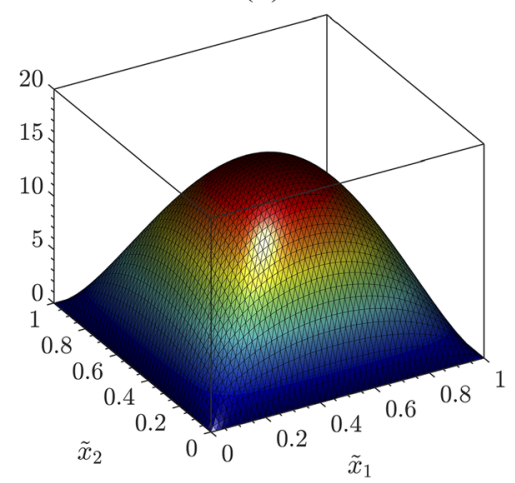

(d)
FIG. 4. Pressure fields developed over smooth surface, unit-square squeeze-films with the following parameters: (a) $\tilde{h}=0.6, M=0$; (b) $\tilde{h}=0.6, M=3$; (c) $\tilde{h}=0.4$, $M=0 ;$ and (d) $\tilde{h}=0.4, M=3$.

\section{RESULTS AND DISCUSSION}

Several numerical examples are conducted to display the model's functionality and robustness. First, we simulate the perfectly smooth, unit-square MHD squeeze-film analyzed by Lin. ${ }^{11}$ This simple example shows the relationship of load-carrying capacity and the Hartmann number, i.e., MHD effects. In the next example, we add surface roughness via the W-M fractal to the previous geometry and investigate its influence on the squeeze-film flow. We then proceed to investigate the effects of several different imposed electric fields on the smooth, unit-square domain. Next, two domains with more interesting shape and topological features are analyzed. Finally, we perform a mesh convergence study to show the convergence rates for both perfectly smooth and rough surfaces with varying fractal dimension. The maximum Hartmann number used in this work is $M=5$, which we consider to be highly practical given that experimental tests performed by Kuzma $^{33}$ featured Hartmann numbers as high as $M \sim 75$ with close agreement to theory.

\section{A. Smooth unit-square with imposed magnetic field}

The majority of studies on MHD squeeze-films use the smooth and finite rectangular plate as the benchmark case; we do the same in this work. The domain is the dimensionless unit-square, $\tilde{\mathbf{x}} \in[0,1]^{2}$. The imposed magnetic field strength $\left\|\mathbf{B}_{0}\right\|$, initial film thickness $h_{0}$, electric conductivity $\sigma$, and fluid viscosity $\eta$ are incorporated into the Hartmann number $M$, which is the varying parameter for this example.

Figure 4 displays the pressure solutions obtained with various film thicknesses and Hartmann numbers, and we observe that the film thickness plays a crucial role in developing the magnitude of the resulting hydrodynamic pressure. This is a well-known phenomenon in squeeze-film flows due to the increased velocity gradients that are present as the filmthickness decreases. The viscous forces are magnified, which in turn increases the balancing pressure forces. We note that the symmetry of the pressure fields is preserved for each case-they only vary in magnitude. Hence, the $\tilde{x}_{2}$ dimension may be suppressed, and the results are plotted along a transect so that each scenario may be clearly compared. This is shown in Fig. 5(a), where the pressure field is extracted along the slice $\tilde{x}_{2}=0.5$. We observe that the magnitude of hydrodynamic pressure is significantly increased for greater Hartmann numbers. As seen in Fig. 5(b), this also results in greater loadcarrying capacity of the squeeze-film, which is computed by (27). The results that have been obtained here are in very good agreement with the analytical solution by Lin. ${ }^{11}$

\section{B. Fractally rough unit-square}

The effect of surface roughness on the flow is modeled with the generalized W-M fractal. Consider a smooth bottom plate and a fractal top plate with film-thickness

$$
\tilde{h}\left(\tilde{x}_{1}, \tilde{x}_{2}\right)=\tilde{h}_{\text {avg }}+\tilde{\zeta}\left(\tilde{x}_{1}, \tilde{x}_{2}\right),
$$

where $\tilde{h}_{\text {avg }}$ represents an average, smooth part and $\tilde{\zeta}\left(\tilde{x}_{1}, \tilde{x}_{2}\right)$ represents the rough, fractal part. A singularity in the solution occurs when $\tilde{h} \leq 0$ (i.e., contact), so we enforce strict positivity of $\tilde{h}$ to ensure contact is avoided. Analyses are performed on four realizations of the W-M fractal, each generated with increasing fractal dimension and random phases 


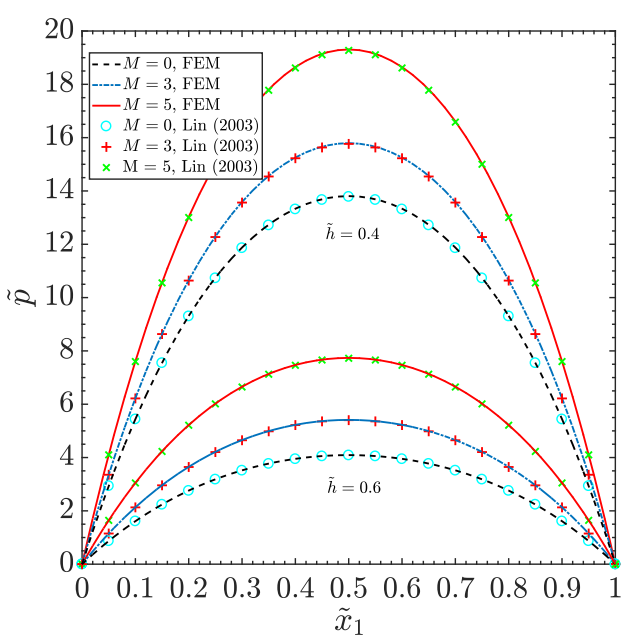

(a)

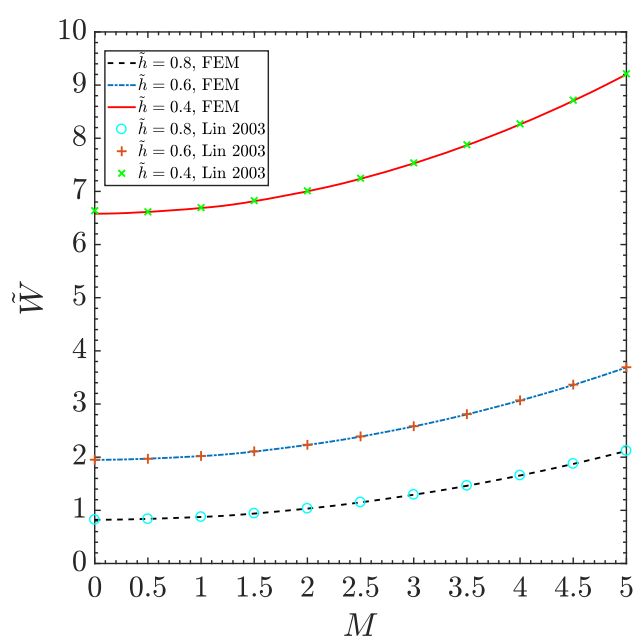

(b)
FIG. 5. (a) Pressure along a slice $\tilde{x}_{2}=0.5$ and (b) load-carrying capacity for various $\tilde{h}$ and $M$ values. $\phi_{m, n} \in[0,2 \pi]$ sampled from a uniform distribution. The triangulated mesh for each surface is shown in Fig. 6 where we assign the names (a) WM1, (b) WM2, (c) WM3, and (d) WM4. Their corresponding fractal dimensions are $D_{H}=2.2,2.4,2.6$, 2.8 , respectively. As expected, the number of asperities per unit of area grows with increasing fractal dimension. Finally, we will not impose any electromagnetic conditions, i.e., $M \rightarrow 0$, in order to investigate the isolated effects of the roughness structure.

Figures 7(a)-7(d) show the resulting pressure field solutions developed over the films characterized by WM1, WM2, WM3, and WM4, respectively. It is evident that the surface features at this length-scale significantly influence the pressure solution and thus the flow field, e.g., see the corresponding velocity fields in Figs. 8(a)-8(d). Our model captures both local and global flow features as a result of particular roughness structures; this is evidenced by the ripples and asymmetries in the pressure solution. The previously mentioned roughness studies that follow Christensen's stochastic approach ${ }^{19}$ solve a statistically averaged governing equation, so the result is an expected pressure field that is always symmetric and does not elucidate the flow perturbations caused by the actual roughness structure. Furthermore, Christensen's approach is based on the principal assumption that the number of asperities per unit area is arbitrarily high, implying that the roughnessinduced flow perturbations are only high frequency ripples

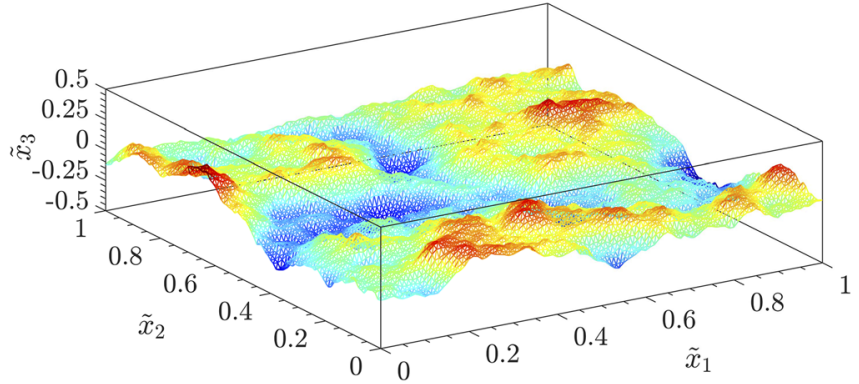

(a)

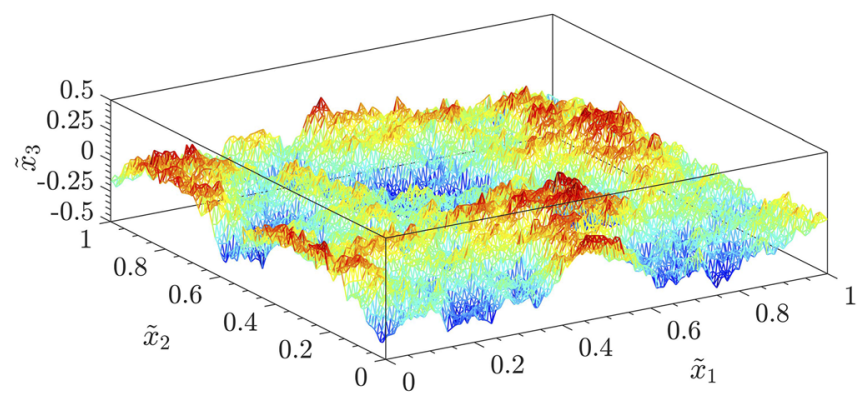

(c)

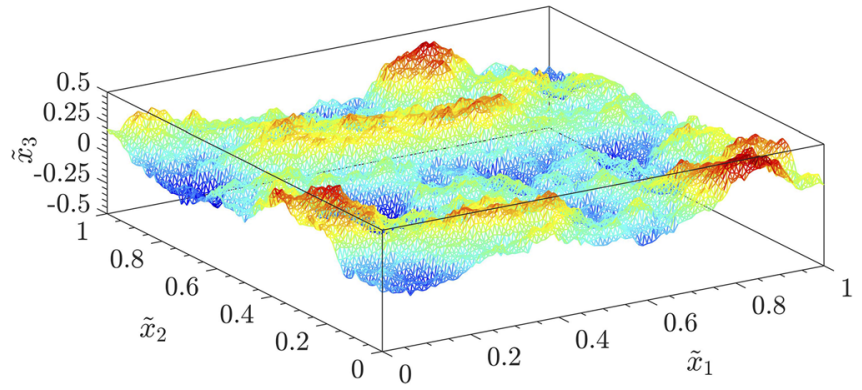

(b)

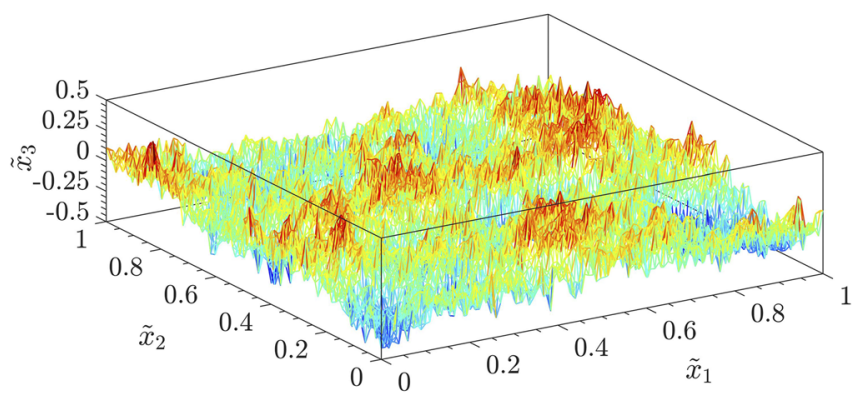

(d)

FIG. 6. Triangulated meshes for different realizations of the W-M fractal referred to as (a) WM1, (b) WM2, (c) WM3, and (d) WM4. Each mesh is composed of 20000 simplex elements. The fractal parameters are $\tilde{\sigma}_{\zeta}^{2}=0.017, \gamma=1.5, m_{\max }=20, \tilde{L}=1$, and $\tilde{L}_{S}=5 \times 10^{-6}$ with uniformly distributed random phases. (a) $D_{H}=2.2$. (b) $D_{H}=2.4$. (c) $D_{H}=2.6$. (d) $D_{H}=2.8$. 


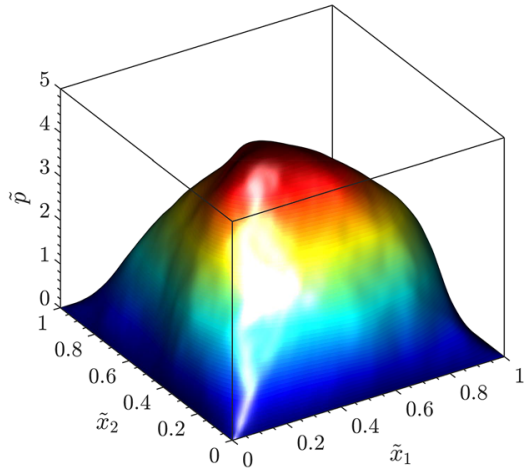

(a)

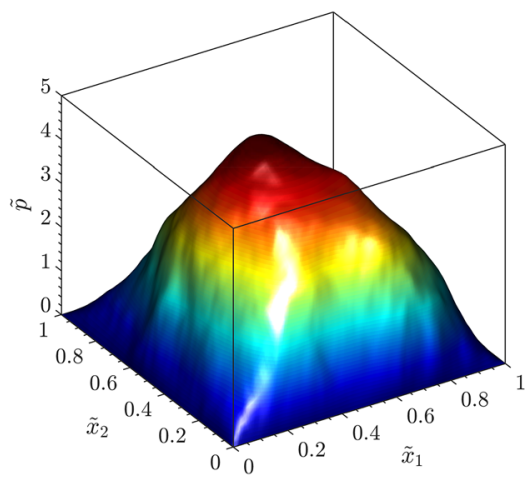

(c)

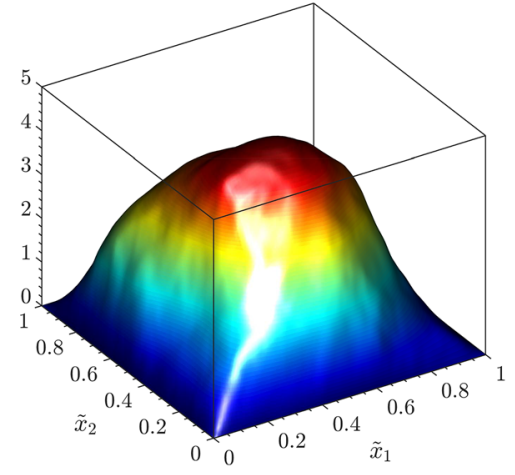

(b)

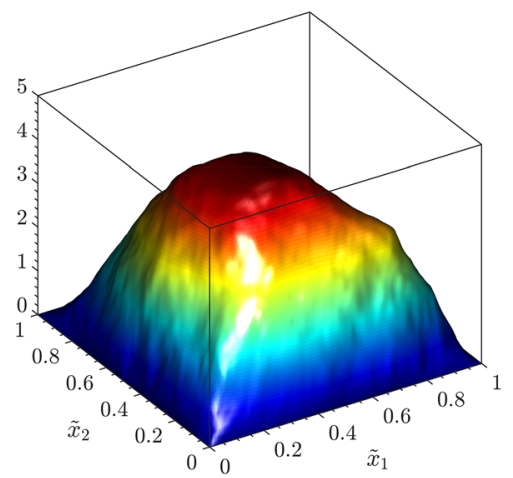

(d)
FIG. 7. Pressure fields for the surfaces WM1, WM2, WM3, and WM4 with initial film thickness $\tilde{h}_{\text {avg }}=0.6$ and Hartmann number $M=0$. (a) WM1. (b) WM2. (c) WM3. (d) WM4. with negligible variance. In other words, fluctuations are assumed perfectly local and statistically uncorrelated, allowing the flow solution and roughness structure to be taken as stochastically independent variables. Our results show that this is generally not the case when modeling the problem with fractal surfaces, which are composed of a wide spectrum of frequencies. The WM4 surface possesses high asperity density characterized by the large fractal dimension, which is

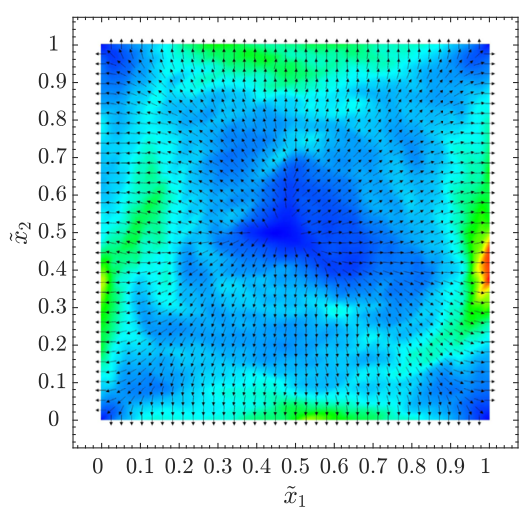

(a)

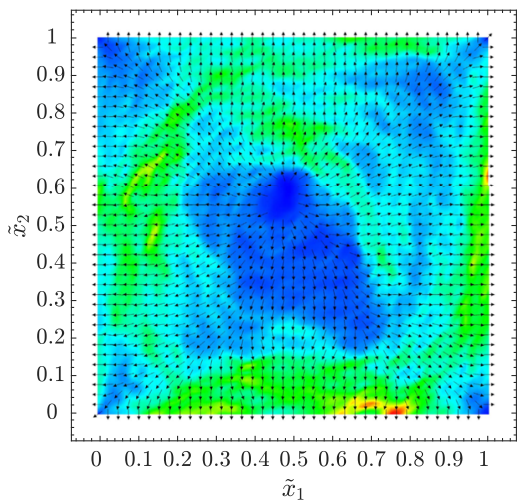

(c)

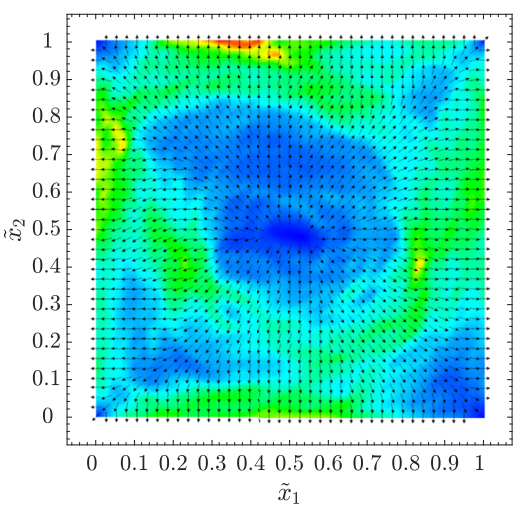

(b)

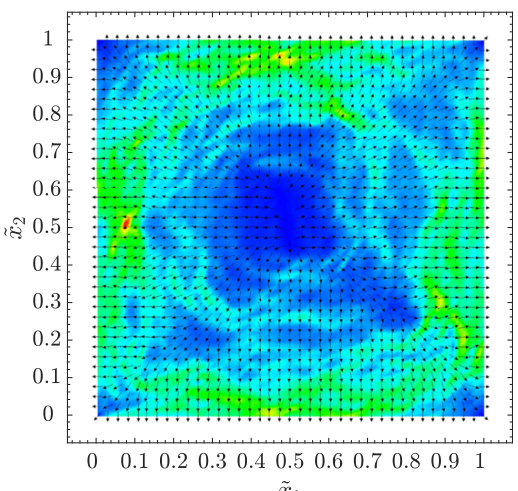

(d)
FIG. 8. Two-dimensional velocity fields where the arrows represent direction-only with the magnitude plotted as the underlying color-map. (a) WM1. (b) WM2. (c) WM3. (d) WM4. 


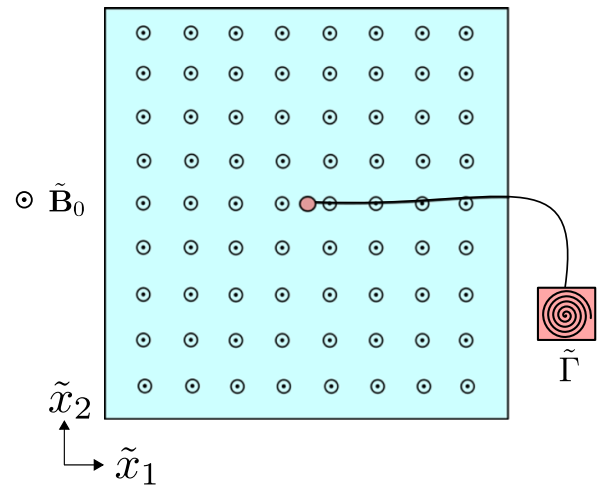

FIG. 9. Smooth unit-square with an imposed magnetic field and electrical current. The rotational electric field is generated by a theoretical device capable of producing a vortex anywhere in the domain with circulation $\tilde{\Gamma}$.

closer to the type of surface that Christensen assumed. Correspondingly, these smaller-amplitude, high-frequency pressure ripples are beginning to emerge. However, even for this case of an over-exaggerated asperity density, we still observe, in addition to high-frequency ripples, significant low-frequency fluctuations that are clearly correlated with the roughness structure.

Christensen only attempted unidirectional roughness profiles, such as transverse and longitudinal directions for long bearings, which may be more amenable to his assumptions. However, others that have used the same approach to investigate isotropic roughness structures have seen varying results. For example, Tonder ${ }^{14,15}$ claimed that roughness-induced pressure fluctuations are purely local and that load-carrying capacity diminishes with increasing surface roughness, while others claimed that the opposite effect is to be expected. ${ }^{16,17}$ We believe, according to these preliminary results, that the flow properties are highly dependent on the roughness structure itself, including both the probability distribution and the power spectrum of the surface; the latter is completely disregarded in the statistical methods derived from Christensen. We are currently employing the model developed here to investigate the isotropic roughness effect in greater detail.

\section{Smooth unit-square with imposed magnetic field and electrical current}

We consider several variations of the setup shown in Fig. 9 to investigate the effect of an injected electrical current. Due to the assumed conductive nature of the fluid, imposing an electric current is alike to supplying an electric field, where the only difference is in the magnitudes of the vectors. The ratio between the magnitude of current density and electric field is the conductivity, dictated by Ohm's law. Since the two are directly related to each other, we use the terms "imposed electric field" and "injected electrical current" synonymously here. Electric fields generated solely with a voltage difference are inherently irrotational (i.e., conservative); therefore, according to our prior discussion, electrostatic fields should have no net effect on the load-carrying capacity of a perfectly smooth squeeze-film. It is thus necessary to introduce a rotational field to dissipate the flow energy, which is analogous to the popular concept of eddy-current braking.

According to Faraday's law, eddy-currents only occur in conjunction with a time-varying magnetic field, which suggests that treatment of the unsteady induction equation is required. While we reserve a full time-dependent simulation for later work, here we retain the quasi-steady assumption by leveraging Maxwell's conclusions from his studies on eddycurrents induced in conducting sheets. ${ }^{34}$ In particular, he found that given an impulsive change in magnetic field (completely localized in time) created by a source outside the conductor, an eddy-current is instantaneously induced that exactly opposes the change in field strength inside the conductor. Then in a transient phase, the current decays due to finite conductivity until the additional field provided by the source diffuses completely into the conductor at steady-state. Disregarding the transient phase, we analyze the time-instant directly following an impulse change of magnetic field, where the eddy-current is present among the initially imposed magnetic field. Because the eddy-current at the initial instant exactly cancels out the change of field strength, the load carrying capacity attained in this configuration is equivalent to the steady-state case where the current has completely decayed and the magnetic field has increased accordingly.

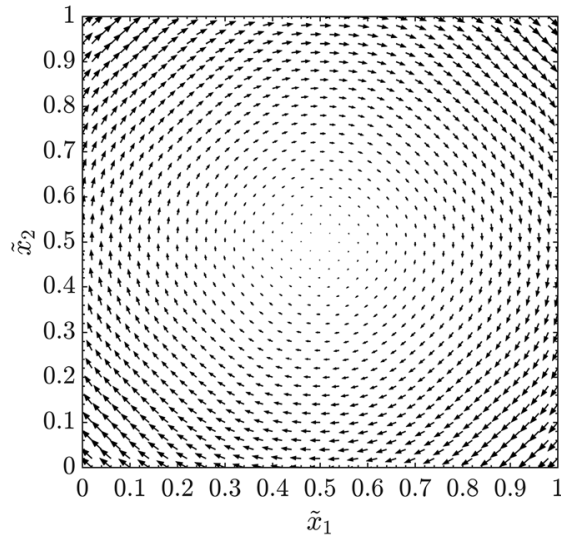

(a)

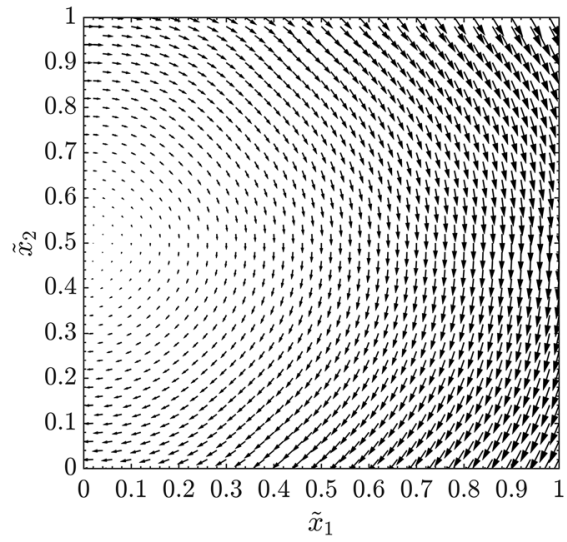

(b)

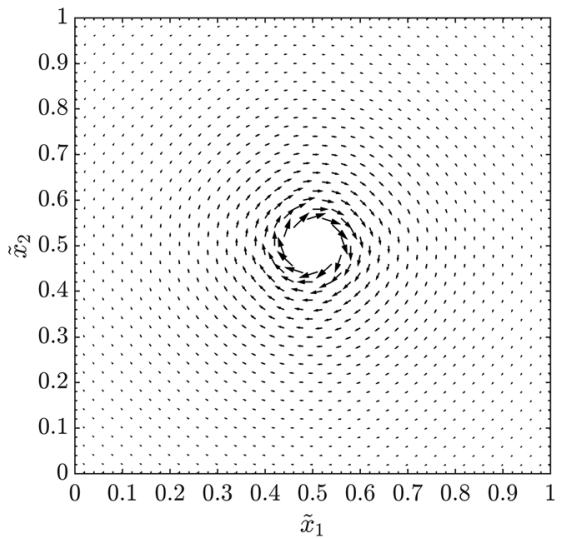

(c)

FIG. 10. Three cases of imposed vortical electric fields. Cases (a) and (b) are Rankine core (forced) vortices with different central locations, and (c) is an irrotational (free) vortex with its singularity removed. (a) VORT1. (b) VORT2. (c) VORT3. 
TABLE I. Input parameters used to generate three vortical electric fields to be imposed into the fluid domain. The vortex strength is denoted by $\tilde{\Gamma}$, and $d\left(\tilde{\mathbf{x}}, \tilde{\mathbf{x}}_{s}\right)=\left\|\tilde{\mathbf{x}}-\tilde{\mathbf{x}}_{S}\right\|_{2}$ is the $\ell^{2}$ distance metric.

\begin{tabular}{llccc}
\hline \hline Case & Type & $\tilde{\psi}$ & $\tilde{\omega}_{e}$ & $\tilde{\mathbf{x}}_{s}$ \\
\hline VORT1 & Rankine core & $\frac{\tilde{\Gamma}}{4 \pi R^{2}} d\left(\tilde{\mathbf{x}}, \tilde{\mathbf{x}}_{s}\right)^{2}$ for $d\left(\tilde{\mathbf{x}}, \tilde{\mathbf{x}}_{s}\right)<R$ & $-\frac{\tilde{\Gamma}}{\pi R^{2}}$ & {$\left[\begin{array}{ll}0.5 & 0.5\end{array}\right]^{T}$} \\
VORT2 & Rankine core & Same as VORT1 & $-\frac{\Gamma}{\pi R^{2}}$ & {$\left[\begin{array}{lll}0.0 & 0.5\end{array}\right]^{T}$} \\
VORT3 & Irrotational & $\frac{\tilde{\Gamma}}{2 \pi} \ln \left(d\left(\tilde{\mathbf{x}}, \tilde{\mathbf{x}}_{s}\right)\right)$ for $d\left(\tilde{\mathbf{x}}, \tilde{\mathbf{x}}_{s}\right) \neq 0$ & 0 & {$\left[\begin{array}{ll}0.5 & 0.5\end{array}\right]^{T}$} \\
\hline \hline
\end{tabular}

For the numerical study, we presume to have access to a theoretical device that is capable of generating precise eddy-currents in the fluid by an impulse change in magnetic field. Such a device could be a solenoid that is spontaneously charged. The current producing device is indicated by the spiraled box in Fig. 9, which we assume produces an electric field following

$$
\tilde{\mathbf{E}}=\mathbf{i}_{2} \tilde{\nabla} \wedge \tilde{\psi},
$$

where $\tilde{\psi}$ is the stream function for the $\mathbf{E}$-field. The rotational behavior of the field is easily quantifiable by its vorticity,

$$
\tilde{\omega}_{e}=-\mathbf{i}_{2}(\tilde{\nabla} \wedge \tilde{\mathbf{E}})=-\tilde{\nabla}^{2} \tilde{\psi} .
$$

We test three different input fields shown in Figs. 10(a)-10(c); these are electric field analogs to popular vortices traditionally found in fluid mechanics. The first case, (a) VORT1, is the forced core of a monopole Rankine vortex, which is often referred to as a rigid-body vortex. The vortex is characterized by strength $\tilde{\Gamma}$ and linearly increasing velocities from zero at the vortex center $\tilde{\mathbf{x}}_{s}$ to a maximum at reference radius $R=1$. The vorticity throughout the core of a Rankine vortex is constant, so we expect this field to only change the magnitude of the induced hydrodynamic pressure and not its general shape. As further evidence of this, the second case tested is another Rankine vortex with the center placed at a different location; see Fig. 10(b) and Table I. The final test case is an irrotational vortex, VORT3, which is shown in Fig. 10(c). Despite its appearance, this "vortex" has no vorticity aside from a singularity at the center. This field is generated with the Green's function solution of (45) and is provided as a counter-example to highlight that an imposed electric field possessing no mathematical vorticity will not influence the load-carrying capacity. We replace the singularity at the center with an interpolated value, which heuristically removes the singular vorticity. The relevant parameters for each of the test cases are compiled in Table I.

The pressure fields are symmetric, so we present the results restricted to the slice $\tilde{x}_{2}=0.5$, which are shown in Fig. 11(a). All three input fields are tested for a range of vortex strengths between $\tilde{\Gamma}=0$ and $\tilde{\Gamma}=15$. As expected when $\tilde{\Gamma}=0$ for all cases, there is no effect from the imposed electric field, leading to the same result as seen in Fig. 5 for corresponding flow parameters. In cases VORT1 and VORT2, we observe a steady increase in the magnitude of the induced pressure as a function of $\tilde{\Gamma}$ and that both fields produce nearly identical results. This is to be expected since, despite their differing locations, both have the same vorticity. For case VORT3, the pressure solution is nearly the same as that of $\tilde{\Gamma}=0$ due to the field being irrotational-the slight deviation is the error introduced when removing the singular value at the center. The load-carrying capacity of the squeeze-film for each case is computed and shown as a function of $\tilde{\Gamma}$ in Fig. 11(b). A linearly increasing trend is observed for cases VORT1 and VORT2, which are nearly identical. The small differences between cases VORT1 and VORT2 can be attributed to numerical error.

\section{Complex geometries and topologies}

We present two examples highlighting the framework's capability to model more complex surface shapes and topologies. The triangulated domains of each are shown in Fig. 12, where case (a) CGT1 is a standard annular disk and (b) CGT2 is a disk with various holes of different shapes. These geometries may represent a realistic design of a squeeze-film damper that is to be used in some mechanical system where such holes are unavoidable due to a design constraint. Obviously, holes and cutouts in the surface permit fluid to more easily flow out of the interface. For this reason, a significant decrease in the magnitude of generated hydrodynamic pressure is to be expected.

The resulting pressure field for each case is shown in Fig. 13, where steep gradients have manifested in order to satisfy the homogeneous boundary conditions for the interior and exterior boundaries. This corresponds to an increase in the flow directed toward the interior holes, which is more clearly seen in the flow-field plots of Figs. 14(a) and 14(b). Due to the axial symmetry of CGT1 and mass conservation, the velocities are greater through the interior cut-out as opposed to the flow out

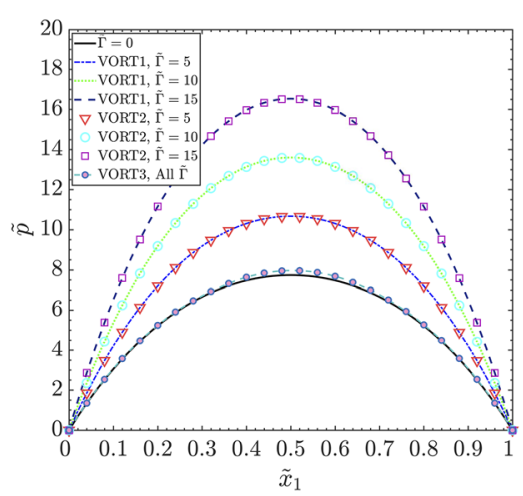

(a)

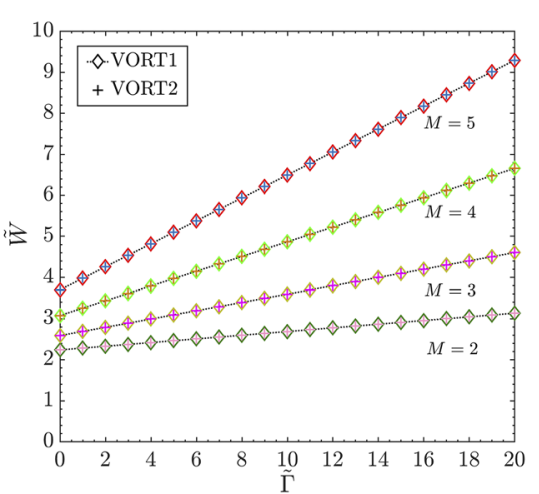

(b)
FIG. 11. (a) Pressure profile along the slice $\tilde{x}_{2}=0.5$ as a result of the different types and strengths of current vortices. (b) Load carrying capacity as a function of vortex strength. The squeeze-film parameters are $\tilde{h}=0.6$, $M=5$, and smooth surfaces. 


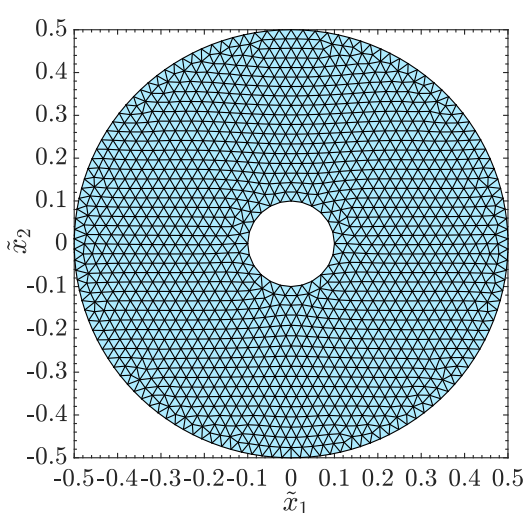

(a)

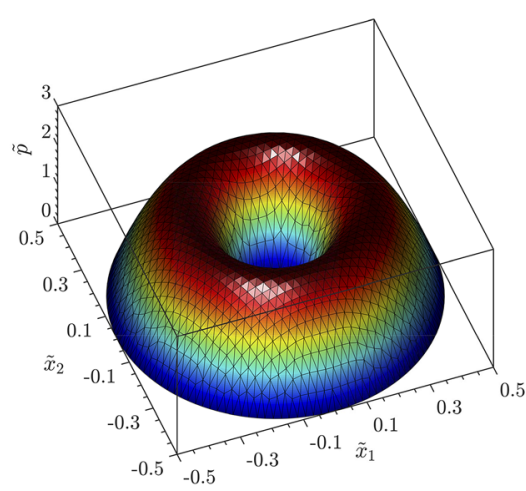

(a)

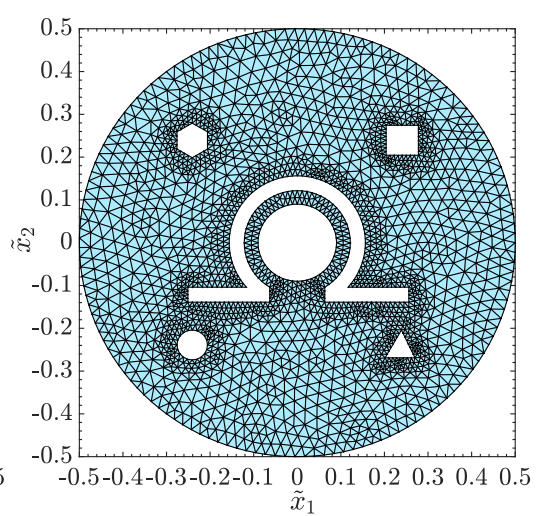

(b)

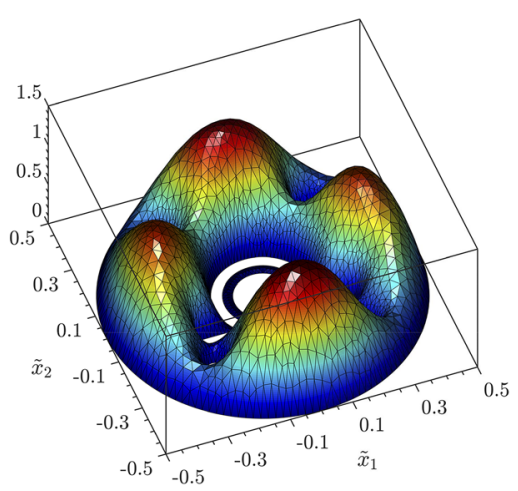

(b)
FIG. 12. (a) Standard annular disk mesh with outer radius $r_{o}=0.5$ and inner radius $r_{i}=0.2$. (b) Annular disk with additional, complex topological features. (a) CGT1. (b) CGT2.
FIG. 13. Pressure fields for the cases (a) CGT1 and (b) CGT2 with squeeze parameters $\tilde{h}=0.6$ and $M=5$. of the exterior boundary. In CGT2, the flow is always directed toward an interior cut-out or toward the exterior boundary. We note the large velocity magnitudes concentrated at several sharp corners of the cutouts; care should be taken here to ensure an adequate mesh refinement is used at these locations.

The load-carrying capacity versus $M$ for both cases is presented in Fig. 15. As expected, $\tilde{W}$ is much smaller for these setups than in previous examples. Furthermore, it is observed that case CGT2 offers significantly less support than CGT1 due to the additional holes in the disk. These two examples show that the geometry and topology of the squeeze-film surface have a substantial influence on the flow and the overall loadcarrying performance of the squeeze-film. The significance of the MHD effects is again clearly apparent; even for modest values of $M$, the load-carrying capacity can be significantly increased. Leveraging this effect could prove extremely valuable for the design of squeeze-film bearings in which geometrical constraints adversely affect the performance of the damper.

\section{E. Mesh convergence}

By definition, a necessary condition for a convergent numerical method is that of consistency, which requires the truncation error of the solution to approach zero as the mesh resolution is increased. While there have been many studies with satisfactory results regarding the convergence properties of finite element methods in general, the convergence of the

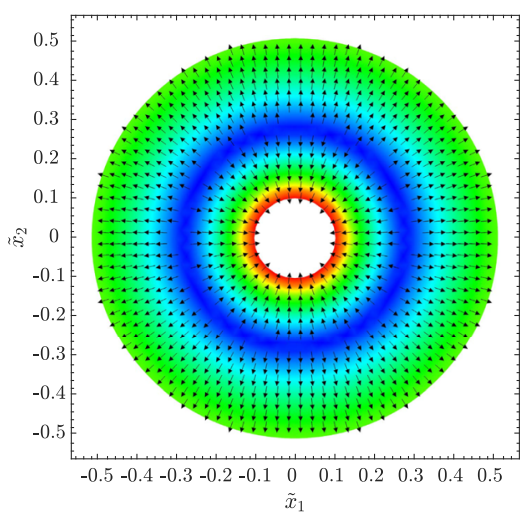

(a)

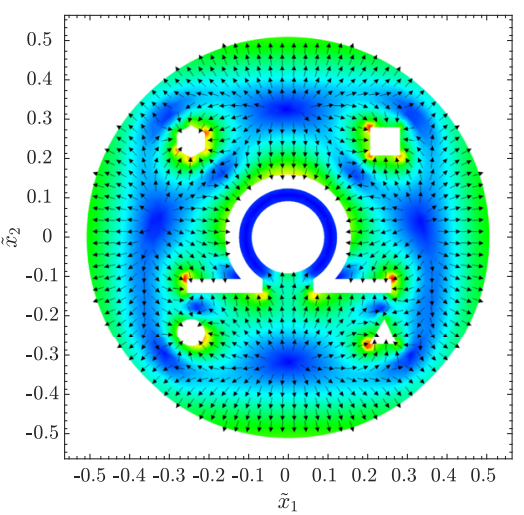

(b)
FIG. 14. Velocity fields for cases (a) CGT1 and (b) CGT2. The vector field is plotted on top of the color-map depicting the velocity magnitude. The size of arrows is not scaled to speed. 


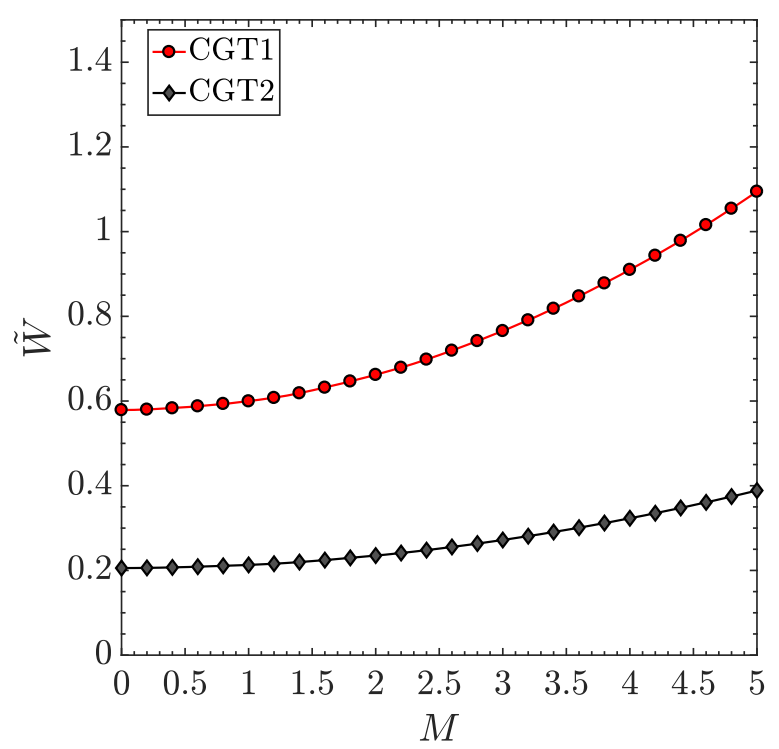

FIG. 15. Load-carrying capacity as a function of Hartmann number for cases CGT1 and CGT2 with an initial film thickness $\tilde{h}=0.6$.

method developed in this paper is complicated by the numerical representation of the rough surface. One of the fundamental advantages of modeling surface roughness with the W-M fractal is that the spectral properties of the surface remain invariant with mesh resolution. In other words, the additional sampling points introduced with a mesh refinement are assigned surface elevations that do not change the power spectrum of the surface. Furthermore, due in part to the fact that the power spectrum approaches zero with increasing frequency according to (29), the numerical representation of the surface converges as the number of sample points (i.e., mesh resolution) is increased. However, the convergence rate for the surface representation depends on the fractal dimension, and we observe that the speed of convergence decreases for surfaces of greater fractal dimension (i.e., roughness). As the solution of the Reynolds equation is immediately dependent on the surface profiles, it is not surprising that the overall convergence rate of the solution is limited by that of the surface representation itself.

We show this by performing a mesh convergence study on the W-M surfaces that were generated in Sec. V B, along with the perfectly smooth cases used in the other numerical examples. The study is carried out by constructing each surface at multiple refinement levels characterized by the parameter $N$, which is the number of rectangular partitions of the domain in each direction. Upon bisecting each of these partitions to create a triangulation, the number of elements in the domain is given by $2 N^{2}$. Error metrics are computed by

$$
\left\|e^{h}\right\|=\left\|p^{h}-p\right\|,
$$

where $p^{h}$ is the numerical solution for a given resolution and $p$ is the numerical solution for the finest resolution. The convergence rate is then determined by

$$
\alpha=\frac{\log \frac{\left\|e^{h / 2}\right\|}{\left\|e^{h}\right\|}}{\log 2},
$$

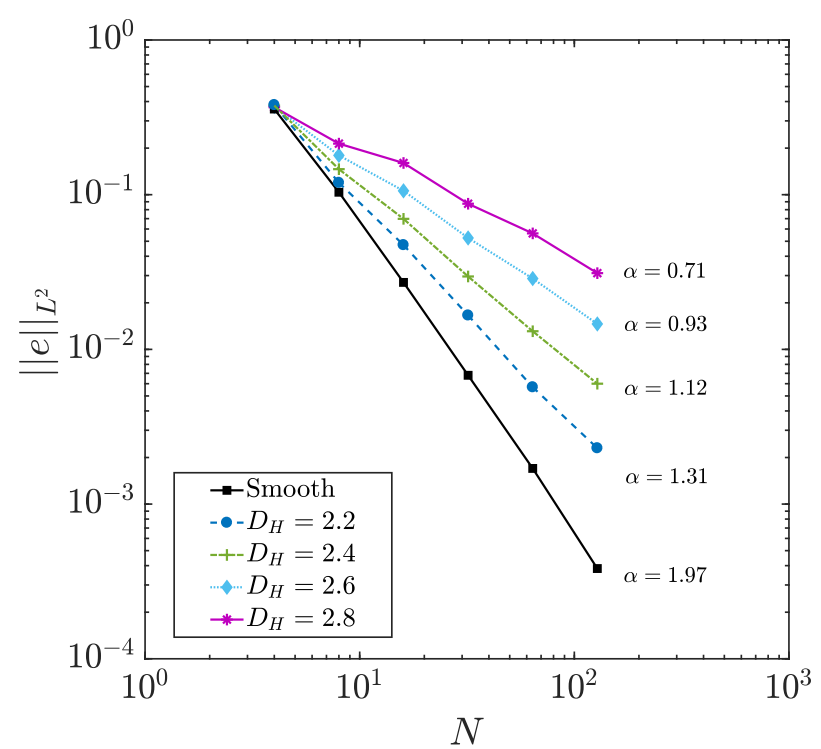

FIG. 16. Numerical errors and convergence rates based on mesh resolution parameter $N$ for perfectly smooth and rough surfaces of varying fractal dimension.

where $e^{h / 2}$ is the numerical error associated with a mesh that is refined by a factor of two.

The convergence results are presented in Fig. 16. As seen from the figure, the convergence rate of the perfectly smooth surfaces is $\alpha \approx 2$, which is well-known in literature for linear finite elements. The rate decreases for increasing fractal dimension, which is to be expected according to the discussion in the previous paragraph. It is clear from the figure that, while all cases ultimately do converge, the convergence rate of the overall method is limited by that of the mathematical representation of the surfaces.

\section{CONCLUSION}

We have developed a robust computational model to investigate various types of squeeze-film flows. In particular, the model is capable of simulating magnetohydrodynamic squeeze-films featuring an electrically conductive fluid that may interact with imposed magnetic and electric fields. This magnetohydrodynamic effect may be used to augment the role of viscous damping - which is usually diminished in high-temperature environments - to increase the squeeze-film performance.

Additionally, the framework is capable of modeling the effects of any general surface topography of the confining boundaries. These input surface profiles may be digitally generated with a wide range of numerical techniques or acquired from the direct measurement of real surfaces. We present the use of the Weierstrass-Mandelbrot fractal to numerically generate extremely realistic, scale-invariant surfaces as inputs for the model. This deterministic approach to modeling surface roughness captures the local flow perturbations caused by any particular surface feature, as well as the global influence that any particular roughness structure has on the squeeze-film performance.

The governing mathematics are presented in a complete manner in the context of geometric algebra, beginning 
with the canonical equations for fluid dynamics (NavierStokes) and electromagnetism (Maxwell). Assuming that a small magnetic-Reynolds number characterizes the flow and employing the common thin-film assumptions, we derive the final form of the magnetohydrodynamic Reynolds equation for squeeze-films. Our derivation is more general than that of others in that both a magnetic and electric field may be imposed. The weak form of the magnetohydrodynamic Reynolds equation is presented and shown to relax smoothness requirements on the squeeze-film surface profile. We implement a Galerkin finite element method to solve the weak form, naturally allowing great flexibility in the geometry and topology of the squeeze-film surfaces to be modeled.

The presented model is capable of quickly analyzing the performance of realistic squeeze-film dampers to be used in mechanical systems. It may also be employed to study various types of squeeze-film flows found in nature. In either application, the model is highly general and not limited to any one domain shape, topography, or topology.

\section{ACKNOWLEDGMENTS}

This material is based upon work supported by the National Science Foundation Graduate Research Fellowship Program under Grant No. 1450681. Any opinions, findings, and conclusions or recommendations expressed in this material are those of the authors and do not necessarily reflect the views of the National Science Foundation.

\section{APPENDIX: OVERVIEW OF GEOMETRIC ALGEBRA/CALCULUS}

The algebra/calculus used in this work is based on the geometric interpretation of the Clifford algebra formalized by Hestenes and Sobczyk, ${ }^{20}$ which enables us to expunge the restriction of the Lorentz force to $\mathbb{R}^{3}$ caused by its cross product definition. We give a short introduction to the algebra here and provide a list of identities that should be sufficient for the unfamiliar reader to make sense of the operations used in this work.

An $n$-dimensional inner-product space $\mathscr{A}_{n}$ induces a $2^{n}$ dimensional vector space $\mathcal{G}\left(\mathscr{A}_{n}\right)$ called the geometric algebra, whose members $A \in \mathcal{G}\left(\mathscr{A}_{n}\right)$ are referred to as multivectors. A general multivector is a linear combination of oriented geometric entities representing the composing subspaces of $\mathscr{A}_{n}$ at every dimension $0 \leq k \leq n$, such as the scalar $(k=0$, point), vector $(k=1$, line), bivector ( $k=2$, plane), and trivector ( $k=3$, volume). Each of these basis objects is said to be of grade $k$ and is referred to as a $k$-blade. If $A$ is a linear combination of only 1-blades, then $A$ is nothing more than a vector; hence, the inducing inner-product space $\mathscr{A}_{n}$ itself is a subspace of the geometric algebra. Consequently, the geometric algebra is an extension of $\mathscr{A}_{n}$ to a space with additional structure.

This additional algebraic structure is needed in order to define a product operation under which multivectors (and hence, vectors) are closed, namely, the geometric product. In other words, the geometric product of any two multivectors $A, B \in \mathcal{G}\left(\mathbb{R}^{n}\right)$ results in another multivector that is also an element of $\mathcal{G}\left(\mathbb{R}^{n}\right)$. The geometric product between two vectors $\mathbf{u}, \mathbf{v} \in \mathbb{R}^{n}$ is

$$
\mathbf{u v}=\mathbf{u} \cdot \mathbf{v}+\mathbf{u} \wedge \mathbf{v},
$$

where $\mathbf{u} \cdot \mathbf{v}$ and $\mathbf{u} \wedge \mathbf{v}$ are, respectively, the inner and outer products. The inner product operates as usual, returning a scalar quantifying the magnitude of the parallel component between the two vectors. The outer product returns a bivector with magnitude equal to the area of the parallelogram formed by the two vectors. Thus, the result of the geometric product between two vectors is a multivector composed of a scalar and bivector component determined by $\mathbf{u} \cdot \mathbf{v}$ and $\mathbf{u} \wedge \mathbf{v}$, respectively. Though the above definitions are manifestly coordinate-free, introducing the standard Euclidean basis for $\mathbb{R}^{n}$ makes the behavior of the product clear. The canonical Euclidean basis is orthonormal, so the geometric product between any two basis vectors results in

$$
\mathbf{e}_{i} \mathbf{e}_{j}= \begin{cases}\mathbf{e}_{i} \wedge \mathbf{e}_{j}, & i<j \\ 1, & i=j . \\ -\mathbf{e}_{j} \wedge \mathbf{e}_{i}, & i>j\end{cases}
$$

This implies that the geometric product between orthonormal vectors is antisymmetric, i.e., $\mathbf{e}_{i} \mathbf{e}_{j}=-\mathbf{e}_{j} \mathbf{e}_{i}$, and that the result is a bivector. Though it is clearly not communicative, the geometric product is associative, allowing us to compose the operation

\begin{tabular}{|c|c|c|c|c|}
\hline Algebra & Grade & Canonical basis & Blade & Geometric entity \\
\hline \multirow[t]{4}{*}{$\mathcal{G}\left(\mathbb{R}^{2}\right)$} & 0 & 1 & Scalar & Point \\
\hline & 1 & $\mathbf{e}_{1} \mathbf{e}_{2}$ & Vector & Line \\
\hline & 2 & $\mathbf{e}_{12}$ & Bivector & Plane \\
\hline & General & $A=\alpha+\sum_{i=1}^{2} a_{i} \mathbf{e}_{i}$ & Pseudoscalar & \\
\hline \multirow[t]{5}{*}{$\mathcal{G}\left(\mathbb{R}^{3}\right)$} & 0 & 1 & Scalar & Point \\
\hline & 1 & $\mathbf{e}_{1} \mathbf{e}_{2} \mathbf{e}_{3}$ & Vector & Line \\
\hline & 2 & $\mathbf{e}_{12} \mathbf{e}_{13} \mathbf{e}_{23}$ & Bivector & Plane \\
\hline & 3 & $\mathbf{e}_{123}$ & Trivector & Volume \\
\hline & \multicolumn{4}{|c|}{ General multivector: $A=\alpha+\sum_{i=1}^{3} a_{i} \mathbf{e}_{i}+\sum_{i=1}^{2} \sum_{\substack{j=2 \\
j>i}}^{3} b_{i j} \mathbf{e}_{i j}+c \mathbf{e}_{123}$} \\
\hline
\end{tabular}

TABLE II. The canonical, Euclidean bases of each grade for the geometric algebras over $\mathbb{R}^{2}$ and $\mathbb{R}^{3}$. 
TABLE III. Pseudoscalar identities in the geometric algebras over $\mathbb{R}^{2}$ and $\mathbb{R}^{3}$.

\begin{tabular}{llrl}
\hline \hline Algebra & \multicolumn{1}{c}{ Identity } & \multicolumn{1}{c}{ Type } \\
\hline $\mathcal{G}\left(\mathbb{R}^{2}\right)$ & Commutation & $\begin{array}{c}\mathbf{a i}_{2}=-\mathbf{i}_{2} \mathbf{a} \\
\left(\mathbf{i}_{2}\right)^{-1}=-\mathbf{i}_{2}\end{array}$ & Pseudovector \\
& Inverse & $\left(\mathbf{i}_{2}\right)^{2}=-1$ & Pseudoscalar \\
& Square & $\mathbf{a} \cdot\left(\mathbf{i}_{2} \mathbf{b}\right)=(\mathbf{a} \wedge \mathbf{b}) \mathbf{i}_{2}$ & Scalar \\
& Inner/outer product & $\mathbf{a} \wedge\left(\mathbf{i}_{2} \mathbf{b}\right)=(\mathbf{a} \cdot \mathbf{b}) \mathbf{i}_{2}$ & Scalar \\
& Commutation: & $\mathbf{a i}_{3}=\mathbf{i}_{3} \mathbf{a}$ & Pseudoscalar \\
& Inverse & $\left(\mathbf{i}_{3}\right)^{-1}=-\mathbf{i}_{3}$ & Bivector \\
& $\left(\mathbf{i}_{3}\right)^{2}=-1$ & Pseudoscalar \\
& Square & $\mathbf{a} \cdot\left(\mathbf{i}_{3} \mathbf{b}\right)=(\mathbf{a} \wedge \mathbf{b}) \mathbf{i}_{3}$ & Scalar \\
& Inner/outer product & $\mathbf{a} \wedge\left(\mathbf{i}_{3} \mathbf{b}\right)=(\mathbf{a} \cdot \mathbf{b}) \mathbf{i}_{3}$ & Pseudovector \\
& & Pseudoscalar \\
\hline \hline
\end{tabular}

TABLE IV. Common geometric algebra/calculus operations in $\mathbb{R}^{2}$ and $\mathbb{R}^{3}$.

\begin{tabular}{|c|c|c|c|c|}
\hline Space & Operation & Geometric form & Vector form ${ }^{a}$ & Component form \\
\hline \multirow[t]{5}{*}{$\mathbb{R}^{2}$} & Cross product & $(\mathbf{a} \wedge \mathbf{b}) \mathbf{i}_{2}$ & $-\langle\mathbf{a} \times \mathbf{b}\rangle_{x_{3}} \mathrm{~b}$ & $-\epsilon_{i j} a_{i} b_{j}^{\mathrm{c}}$ \\
\hline & Scalar triple product & $\mathbf{a} \wedge \mathbf{b} \wedge \mathbf{c}$ & $-\mathbf{a} \cdot(\mathbf{b} \times \mathbf{c})$ & 0 \\
\hline & Vector triple product & $\mathbf{a} \cdot(\mathbf{b} \wedge \mathbf{c})$ & $-\mathbf{a} \times(\mathbf{b} \times \mathbf{c})$ & $\left(b_{1} c_{2}-b_{2} c_{1}\right) \epsilon_{i j} a_{i} \mathbf{e}_{j}$ \\
\hline & Scalar curl & $(\nabla \wedge \mathbf{a}) \mathbf{i}_{2}$ & $-\langle\nabla \times \mathbf{a}\rangle_{x_{3}}$ & $-\epsilon_{i j} \partial_{i} a_{j}$ \\
\hline & Divergence & $\left(\nabla \wedge\left(\mathbf{i}_{2} \mathbf{a}\right)\right) \mathbf{i}_{2}$ & $\nabla \cdot \mathbf{a}$ & $\partial_{j} a_{j}$ \\
\hline \multirow[t]{5}{*}{$\mathbb{R}^{3}$} & Cross product & $(\mathbf{a} \wedge \mathbf{b}) \mathbf{i}_{3}$ & $-(\mathbf{a} \times \mathbf{b})$ & $-\epsilon_{i j k} a_{j} b_{k} \mathbf{e}_{i}$ \\
\hline & Scalar triple product & $(\mathbf{a} \wedge \mathbf{b} \wedge \mathbf{c}) \mathbf{i}_{3}$ & $-\mathbf{a} \cdot(\mathbf{b} \times \mathbf{c})$ & $-\epsilon_{i j k} a_{i} b_{j} c_{k}$ \\
\hline & Vector triple product & $\mathbf{a} \cdot(\mathbf{b} \wedge \mathbf{c})$ & $-\mathbf{a} \times(\mathbf{b} \times \mathbf{c})$ & $-\left(b_{i} a_{j} c_{j}-c_{i} a_{j} b_{j}\right) \mathbf{e}_{i}$ \\
\hline & Curl & $(\nabla \wedge \mathbf{a}) \mathbf{i}_{3}$ & $-\nabla \times \mathbf{a}$ & $-\epsilon_{i j k} \partial_{j} a_{k} \mathbf{e}_{i}$ \\
\hline & Divergence & $\left(\nabla \wedge\left(\mathbf{i}_{3} \mathbf{a}\right)\right) \mathbf{i}_{3}$ & $-\nabla \cdot \mathbf{a}$ & $-\partial_{j} a_{j}$ \\
\hline
\end{tabular}

${ }^{\mathrm{a}}$ The cross operations in $\mathbb{R}^{2}$ are not rigorously defined - the vector forms given here are merely symbolic.

${ }^{\mathrm{b}}$ The operation $\langle\cdot\rangle_{x_{3}}$ indicates the selection of the scalar component in the (fictitious) $x_{3}$ direction.

${ }^{\mathrm{c}} \epsilon_{i j}$ and $\epsilon_{i j k}$ are the Levi-Civita symbols. Einstein summation is implied over repeated indices.

between bases of different grades; for example, the geometric product between the vector $\mathbf{e}_{2}$ and the trivector $\mathbf{e}_{1} \mathbf{e}_{2} \mathbf{e}_{3}$ can be viewed as the composed product of four vectors, i.e.,

$$
\mathbf{e}_{2}\left(\mathbf{e}_{1} \mathbf{e}_{2} \mathbf{e}_{3}\right)=\left(\mathbf{e}_{2} \mathbf{e}_{1}\right) \mathbf{e}_{2} \mathbf{e}_{3}=-\mathbf{e}_{1}\left(\mathbf{e}_{2} \mathbf{e}_{2}\right) \mathbf{e}_{3}=-\mathbf{e}_{1} \mathbf{e}_{3} \text {. }
$$

The n-blade in an algebra $\mathcal{G}\left(\mathscr{A}^{n}\right)$, for example, the bivector $\mathbf{e}_{1} \mathbf{e}_{2} \in \mathcal{G}\left(\mathbb{R}^{2}\right)$, is referred to as a pseudoscalar and denoted by the symbol $\mathbf{i}_{n}$. The pseudoscalar has important and useful properties, perhaps the most captivating of which is its association with the complex number $i=\sqrt{-1}$. This is brought to light using (A2),

$$
\left(\mathbf{i}_{2}\right)^{2}=\left(\mathbf{e}_{1} \mathbf{e}_{2}\right)\left(\mathbf{e}_{1} \mathbf{e}_{2}\right)=-\mathbf{e}_{2}\left(\mathbf{e}_{1} \mathbf{e}_{1}\right) \mathbf{e}_{2}=-\mathbf{e}_{2} \mathbf{e}_{2}=-1 .
$$

Thereupon, we have revealed that the geometric algebra not only naturally defines the complex numbers but also attaches to them a geometric meaning. For example, the complex numbers $z=a+b i \in \mathbb{Z}$ are isomorphic to the multivectors $A=\alpha+\beta \mathbf{i}_{2} \in \mathcal{G}\left(\mathbb{R}^{2}\right)$, where $\mathbf{i}_{2}$ represents the $\mathbf{e}_{1} \mathbf{e}_{2}$ plane. Even further, the geometric algebra does the same with the quaternions $q=a+b i+c j+d k \in \mathbb{H}$, which are isomorphic to the multivectors $A=\alpha+\beta \mathbf{e}_{3} \mathbf{e}_{2}+\delta \mathbf{e}_{1} \mathbf{e}_{3}+\gamma \mathbf{e}_{2} \mathbf{e}_{1}$. While this brief introduction only scratches the surface of geometric algebra, it is all that is needed to form the operations used in this work. All of the identities that we use are presented in the following tables. Table II lays out the canonical bases for the algebras over the Euclidean spaces $\mathbb{R}^{2}$ and $\mathbb{R}^{3}$. Next, Table III gives the common identities of pseudoscalar operations - in particular, how the pseudoscalar can be used to transform between the inner and outer products. Finally, Table IV compares popular vector algebra/calculus operations to those in geometric algebra/calculus, as well the component form of each operation. Most importantly, we note that this table contains the generalization of the cross product to $\mathbb{R}^{2}$, which is what was ultimately desired.

${ }^{1}$ F. Pan, J. Kubby, E. Peeters, A. T. Tran, and S. Mukherjee, "Squeeze film damping effect on the dynamic response of a MEMS torsion mirror," J. Micromech. Microeng. 8, 200 (1998).

${ }^{2}$ M. Bao and H. Yang, "Squeeze film air damping in MEMS," Sens. Actuators, A 136, 3-27 (2007).

${ }^{3}$ J. B. Starr, "Squeeze-film damping in solid-state accelerometers," in IEEE 4th Technical Digest on Solid-State Sensor and Actuator Workshop (IEEE, 1990), pp. 44-47.

${ }^{4}$ F. Y. Zeidan, L. San Andres, and J. M. Vance, "Design and application of squeeze film dampers in rotating machinery," in Proceedings of the 25th Turbomachinery Symposium (Texas A\&M University, 1996), pp. 169-188.

${ }^{5} \mathrm{O}$. Reynolds, "On the theory of lubrication and its application to Mr. Beauchamp tower's experiments, including an experimental determination of the viscosity of olive oil," Proc. R. Soc. London 40, 191-203 (1886).

${ }^{6}$ T. Sasada, Y. Kurosaki, K. Honda, and K. Kamijo, "MHD journal bearing in a magnetic field perpendicular to its axis: 1st report, analysis of an infinitely long bearing," Bull. JSME 17, 1645-1651 (1974).

${ }^{7}$ J.-R. Lin, "Magneto-hydrodynamic squeeze film characteristics between annular disks," Ind. Lubr. Tribol. 53, 66-71 (2001). 
${ }^{8}$ M. Ausloos and D. H. Berman, "A multivariate Weierstrass-Mandelbrot function," Proc. R. Soc. A 400, 331-350 (1985).

${ }^{9}$ M. Faraday and T. Martin, Faraday's Diary: December 28, 1831-January 13, 1832 (G. Bell and Sons, Ltd., 1936), Vol. 1, pp. 409-413.

${ }^{10}$ O. M. Stuetzer, "Magnetohydrodynamics and electrohydrodynamics," Phys. Fluids 5, 534-544 (1962).

${ }^{11}$ J.-R. Lin, "Magneto-hydrodynamic squeeze film characteristics for finite rectangular plates," Ind. Lubr. Tribol. 55, 84-89 (2003).

${ }^{12}$ N. M. Bujurke and R. B. Kudenatti, "MHD lubrication flow between rough rectangular plates," Fluid Dyn. Res. 39, 334-345 (2007).

${ }^{13}$ N. M. Bujurke, N. B. Naduvinamani, and D. P. Basti, "Effect of surface roughness on magnetohydrodynamic squeeze film characteristics between finite rectangular plates," Tribol. Int. 44, 916-921 (2011).

${ }^{14} \mathrm{~K}$. Tonder, "Lubrication of surfaces having area-distributed isotropic roughness," J. Lubr. Technol. 99, 323-328 (1977).

${ }^{15} \mathrm{~K}$. Tonder, "Simulation of the lubrication of isotropically rough surfaces," ASLE Trans. 23, 326-333 (1980).

${ }^{16} \mathrm{H}$. Elrod, "A review of theories for the fluid dynamics effects of roughness of laminar lubrication films," Technical Report No. 27, Columbia University, New York, 1977.

${ }^{17}$ N. Patir and H. Cheng, "An average flow model for determining effects of three-dimensional roughness on partial hydrodynamic lubrication," J. Lubr. Technol. 100, 12-17 (1978).

${ }^{18}$ J. Tripp, "Surface roughness effects in hydrodynamic lubrication: The flow factor method," J. Lubr. Technol. 105, 458-463 (1982).

${ }^{19}$ H. Christensen, "Stochastic models for hydrodynamic lubrication of rough surfaces," Proc. - Inst. Mech. Eng. 184, 1013-1026 (1969).

${ }^{20}$ D. Hestenes and G. Sobczyk, Clifford Algebra to Geometric Calculus: A Unified Language for Mathematics and Physics (Reidel, 1984).
${ }^{21}$ A. Sherman and G. W. Sutton, Engineering Magnetohydrodynamics (McGraw-Hill, 1965)

${ }^{22}$ P. A. Davidson, An Introduction to Magnetohydrodynamics (Cambridge University Press, 2001), Vol. 25.

${ }^{23}$ H. Alfvén, "Existence of electromagnetic-hydrodynamic waves," Nature 150, 405-406 (1942).

${ }^{24}$ H. G. Elrod, "A derivation of the basic equations for hydrodynamic lubrication with a fluid having constant properties," Q. Appl. Math. 17, 349-359 (1960).

${ }^{25}$ W. E. Langlois, "Isothermal squeeze films," Q. Appl. Math. 20, 131-150 (1962).

${ }^{26}$ U. Müller and L. Bühler, Magnetofluiddynamics in Channels and Containers (Springer Science \& Business Media, 2013).

${ }^{27}$ P. R. Nayak, Random Process Model of Rough Surfaces (ASME, 1971).

${ }^{28}$ R. S. Sayles and T. R. Thomas, "Surface topography as a nonstationary random process," Nature 271, 431-434 (1978).

${ }^{29}$ A. Majumdar and C. L. Tien, "Fractal characterization and simulation of rough surfaces," Wear 136, 313-327 (1990).

${ }^{30}$ R. Badii and A. Politi, "Hausdorff dimension and uniformity factor of strange attractors," Phys. Rev. Lett. 52, 1661 (1984).

${ }^{31}$ M. Berry and Z. Lewis, "On the Weierstrass-Mandelbrot fractal function," Proc. R. Soc. London, Ser. A 370, 459-484 (1980).

${ }^{32}$ W. Yan and K. Komvopoulos, "Contact analysis of elastic-plastic fractal surfaces,” J. Appl. Phys. 84, 3617 (1998).

${ }^{33}$ D. Kuzma, "The magnetohydrodynamic squeeze film," J. Fluid Mech. 19, 395-400 (1964).

${ }^{34}$ W. Saslow, "Maxwell's theory of eddy currents in thin conducting sheets, and applications to electromagnetic shielding and MAGLEV," Am. J. Phys. 60, 693-711 (1992) 